\title{
Die Anfänge der Militärischen Laserforschung und Lasertechnik in der Bundesrepublik Deutschland im Zeitalter des Kalten Krieges
}

\author{
Helmuth Albrecht
}

The Early Years of Military Laser Research and Technology in the Federal Republic of Germany During the Cold War

The invention of the laser in 1960 and the innovation process of laser technology during the following years coincided with the dramatic increase of the East-West-conflict during the 1960s - the peak of the so-called Cold War after the erection of the Berlin Wall in 1961. The predictable features of the new device, not only for experimental sciences, but also for technical and military applications, led instantly to a laser hype all over the world. Military funding and research played a major part in this development. Especially in the United States military laser research and development played an important role in the formation of Cold War sciences. The European allies followed this example to a certain degree, but their specific national environments led to quite different solutions and results. This article describes and analyzes the special features and background of this development for the Federal Republic of Germany in the area of conflict between science, politics and industry from 1960 to the early 1970s.

Keywords: Laser History, Military Laser Research, Cold War, Federal Republic of Germany

Schlüsse/wörter: Geschichte des Lasers, Militärische Laserforschung, Kalter Krieg, Bundesrepublik Deutschland

Paul Forman charakterisierte 1992 in seinem Aufsatz „Inventing the Maser in Postwar America“in der Zeitschrift Osiris den Maser $^{1}$ als „an artifact of American cold War culture“ (Forman 1992: 131). Weiter führte er dazu aus: "Yet as a device the maser was an expression not of the traditional goals of physical research, but of an orientation corresponding to the new terms of social integration of Americas physics. That is to say, it was an expression of the specific needs of the U.S. military, now the principal sponsor of the physical research enterprise" (Forman 1992: 134). Forman verglich in seinem inzwischen als klassisch geltenden Aufsatz Maser und Laser $^{2}$ mit dem Transistor und kam dabei zu dem Schluss: „But while the transistor truly increased the ,size' of the solid-state physics enterprise, it had only a small part in creating the 
conceptual categories basic to the field. The historical meaning of the maser and laser is greater, even if the size of the enterprise that they spawned is not" (Forman 1992: 134).

Inventions- und Innovationsgeschichte von sowohl Maser als auch Laser verdeutlichen Forman zufolge paradigmatisch den Wandlungsprozess innerhalb der physikalischen Forschung in den USA nach dem Zweiten Weltkrieg. Das bislang durch Kategorien wie „Offenheit", „Kooperation“ oder „Grundlagenforschung“ bestimmte Selbstverständnis vor allem der universitären Forscher begann sich im Zeichen zunehmender industrieller oder militärischer Anwendungen und den damit verbundenen Patentierungen beziehungsweise einer mit der nationalen Sicherheit begründeten Geheimhaltung $\mathrm{zu}$ wandeln. Das den traditionellen Wissenschaftsbetrieb kennzeichnende Spannungsverhältnis zwischen einer Ideologie der Kooperation auf der einen und einer Realität des Wettbewerbs auf der anderen Seite wurde dadurch, so Forman, um weitere Gegensätze wie persönliches versus nationales Interesse oder Grundlagenforschung versus angewandte Forschung verschärft. Mit ihren wissenschaftlich-technischen Anwendungen versprachen der Maser und in noch viel größerem Umfang der Laser den Physikern und ihren Forschungsinstitutionen nicht nur wissenschaftliche Ehrungen, neue Erkenntnisperspektiven und finanzielle Mittel, sondern der Industrie und dem Staat beziehungsweise seinen Militärs bislang nur erträumte praktische Anwendungen, finanzielle Gewinne und politische wie militärische Machtpotentiale. Zumindest in den USA wurde dies, wie neben Forman auch die Forschungen von Lisa Bromberg oder Robert W. Seidel gezeigt haben, zum idealen Nährboden für die Maser- und Laserforschung (Forman 1992; Bromberg 1991; Seidel 1987; Seidel 1988).

Forman stellte mit seinem Aufsatz von 1992 die Entwicklung des Masers beziehungsweise Lasers in den größeren Kontext der weltweiten Debatte zur Cold War Science, in der er für die USA ganz allgemein einen immer größeren Einfluss nationaler Sicherheitsaspekte auf die Entwicklung der Nachkriegsphysik konstatierte (Forman 1987). Auch wenn das enge Verhältnis zwischen Wissenschaft und Militär im Zeitalter des Kalten Krieges in den Jahren von 1947 bis 1991 in der nunmehr seit drei Jahrzehnten andauernden Debatte um Cold War Sciencenicht grundsätzlich infrage worden gestellt ist, so ist doch inzwischen Kritik an einer zu einseitigen Betonung des Einflusses der Militärs auf die Entwicklung der Wissenschaften in diesem Zeitraum laut geworden. In ihrem einleitenden, die Entwicklung und den aktuellen Forschungsstand der Cold War ScienceDebatte reflektierenden Beitrag in dem Sammelband Perspectives on Cold War Science in Small European States (2013) weisen Matthias Heymann und Janet Martin-Nielsen zwanzigJahre nach Forman darauf hin (vgl. Heymann \& Martin-Nielsen 2013), dass der Einfluss anderer Faktoren auf die Entwicklung der Wissenschaften während des Kalten Krieges vielfach unterschätzt worden sei. So hätten jüngere Forschungen gezeigt, dass 
militärische Auflagen zur Geheimhaltung der Forschung durch den weiter bestehenden Einfluss von Normen einer "freien“ und „offenen“ Wissenschaft unterlaufen worden sind. Auch lasse sich das Beispiel der USA nicht so ohne weiteres auf andere Staaten übertragen. In Westeuropa sei zwar nach dem Vorbild der USA der Sektor der staatlich finanzierten Forschung nach 1945 ebenfalls signifikant ausgebaut worden, aber eben doch mit erheblichen Unterschieden gegenüber dem großen Vorbild: Militärische Forschung und Entwicklung expandierten vor allem in Großbritannien, Frankreich und Schweden, aber auf einem wesentlich niedrigeren Niveau als in den USA. Die Forschungsförderung für militärische Zwecke war in Europa zudem vor allem projekt- und anwendungsorientiert und kaum auf Grundlagenforschung gerichtet. Sie fand in erster Linie in Industrieunternehmen beziehungsweise vornehmlich auf zivile Forschung spezialisierten Forschungseinrichtungen und kaum an Universitäten statt, was dazu führte, dass sie öffentlich kaum sichtbar war und damit lange Zeit nach Umfang und Bedeutung unterschätzt wurde (Heymann \& Martin-Nielsen 2013: 227 f.). Die Geheimhaltung militärischer Forschung diente in Europa nicht nur der nationalen Sicherheit, sondern vielfach auch der Vermeidung öffentlicher Debatten in einer nach zwei Weltkriegen sich in weiten Teilen als international, friedfertig und antimilitaristisch verstehenden Gesellschaft und Wissenschaft. Hinzu kam eine auf wissenschaftliche und technologische Autonomie und Unabhängigkeit vom großen Vorbild und zugleich Rivalen USA zielende Tendenz, die Anforderungen im Bereich der nationalen Sicherheit und Verteidigung gegenüber einer aggressiv auftretenden UdSSR überlagerte. Dies führte zu erheblichen Diskrepanzen im Bereich der Cold War Science nicht nur gegenüber den USA, sondern auch zwischen kleinen und großen Staaten, Demokratien und Diktaturen oder NATO-Mitgliedern und neutralen Staaten in Europa selbst.

Heymann und Martin-Nielsen weisen darauf hin, dass bis heute unser Kenntnisstand über Cold War Science und den militärisch-industriell-wissenschaftlichen Komplex in Europa wesentlich geringer ist als im Fall der USA (Heymann \& Martin-Nielsen 2013: 226). AufGrundlage dieser Erkenntnis und ihrer Analyse der Cold War Science-Debatte leiten sie einige grundsätzliche Fragen ab, die nach Ansicht des Autors nicht nur für die Analyse der Entwicklung in kleinen westeuropäischen Staaten von Bedeutung sind: Wie ordnen sich die europäischen Staaten in das bestehende Bild der Cold War Science ein? Welche Rolle spielten Souveränitätsfragen gegenüber den USA? Und welche Nuancen müssen aus europäischer Sicht den aktuellen Konzepten der Koproduktion von Wissen sowie der im gegenseitigen Einvernehmen bestehenden Hegemonie in den europäisch-amerikanischen Wissenschaftsbeziehungen während des Kalten Krieges hinzugefügt werden (Heymann \& Martin-Nielsen 2013: 231)? 
Am Beispiel der beginnenden militärischen Laserforschung und Lasertechnik in der Bundesrepublik Deutschland in den 1960er Jahren sollen im vorliegenden Aufsatz Antworten auf diese grundsätzlichen Fragen gegeben werden. Dazu werden zwei weitere, bereits 2001 von David A. Hounshell konstatierte zentrale Fragen der Debatte um Cold War Science aufgegriffen: die Fragen nach der Produktion von Wissen sowie die Frage nach der Produktion von technischen Gütern während des Kalten Krieges (vgl. Hounshell 2001: 290). Mit anderen Worten:Im Folgenden soll erörtert werden, ob in der Bundesrepublik durch den Einfluss des Militärs beziehungsweise durch Aspekte der nationalen Sicherheit die wissenschaftlichen Forschungen zum Laser einerseits und die Leistungs- und Wettbewerbsfähigkeit von im Laserbereich aktiven Unternehmen andererseits gefördert oder behindert wurden, Hounshells Aufforderung zum „rethinking science and technology in the Cold War" unter Einbeziehung einer möglichst großen Bandbreite von Analyseeinheiten in die Untersuchung - der einzelnen Wissenschaftler, der wissenschaftlichen Institutionen, der wissenschaftlichen Disziplinen, der wissenschaftlichen Organisationen sowie der Artefakte von Wissenschaft und Technik (Hounshell 2001: 291) -soll dabei exemplarisch am Beispiel der Rolle des Bundesministeriums für Verteidigung (BMVg) sowie des früh in der militärischen Laserforschung aktiven Unternehmens Carl Zeiss in Oberkochen nachgegangen werden.

Bleibt abschließend noch die Frage nach der Wahl des zeitlichen Untersuchungshorizontes der 1960er Jahre zu klären. Die erstmalige Realisierung eines Lasereffektes in den USA macht das Jahr 1960 zum naheliegenden Ausgangspunkt der Untersuchung, auch wenn zumindest für die zivile bundesdeutsche Forschung im Bereich der Quantenelektronik mit den um 1956/1958 aufgenommenen Forschungen zur Hochfrequenzphysik und -technik sowie zum Maser eine gewisse, aufgrund der bis 1955 geltenden alliierten Forschungsbeschränkungen allerdings nur sehr kurze Vorlaufzeit zu konstatieren ist (vgl. Albrecht \& Helmuth 1997: 44-46). Den Endpunkt der Untersuchung bildet die Wende von den späten 1960er zu den frühen 1970er Jahren, die sowohl in den USA als auch in Europa durch einen allgemeinen Rückgang in der Finanzierung militärischer Forschung und Entwicklung sowie eine stärkere Fokussierung auf zivile Forschungsprojekte gekennzeichnet war (Heymann \& Martin-Nielsen 2013: 228). Im Bereich der bundesdeutschen Laserforschung und -entwicklung zeichnete sich Ende der 1960er Jahre eine deutliche Ernüchterung bei den noch zu Beginn des Jahrzehnts überaus großen Erwartungen hinsichtlich einer Kommerzialisierung der neuen Technologie ab. Siemens, das führende deutsche Unternehmen im Bereich der Laserforschung und -entwicklung, reduzierte daher 1970 drastisch seine Ausgaben für diesen Bereich (Fritsch \& Echalar 2015). Um 1970 war die Pionierzeit der Laserentwicklung nicht nur in Deutschland beendet. Die Inventions- und in weiten Teilen auch die Innovationsphase der Lasertechnik 
waren in vielen Anwendungsbereichen abgeschlossen. Allein die Diffusionsphase, das heißt die Markteinführung und Marktdurchsetzung wollte noch nicht so recht gelingen. In zahlreichen Anwendungsbereichen erfolgte ein Durchbruch der Lasertechnik erst in den späten 1970er oder gar erst in 1980er Jahren, wie etwa das Beispiel der Materialbearbeitung zeigt (Fabian 2012). Wichtige Weichenstellungen dafür fanden jedoch sowohl in der zivilen als auch in der militärischen Laserforschung und Lasertechnik bereits in den 1960er Jahren statt.

\section{Der Laser als zivile und militärische Schlüsseltechnologie}

With the advent of optical maser, man's control of light has reached an entirely new level. Indeed, one of the most exciting prospects for workers in the field is that this new order of control will open up uses for light that are as yet undreamed of (Schawlow 1961: 61).

Als der amerikanische Physiker und spätere Nobelpreisträger Arthur L. Schawlow $^{3}$ im Juni 1961 diese enthusiastischen Worte niederschrieb, war der von ihm gefeierte "Optische Maser“ oder auch „Laser“, wie man ihn bald darauf allgemein nennen sollte, gerade ein Jahr alt geworden. Die erstmalige Realisierung eines Lasereffekts in einem Rubinkristall sowie wenig später in einem Helium-Neon-Gasgemisch durch US-Physiker im Sommer und Herbst 1960 löste eine weltweite Lasereuphorie in der Forschung sowie bei der Suche nach praktischen Anwendungen in Wissenschaft und Technik aus. Das USNachrichtenmagazin Time feierte 1961den Laser als „one of the most revolutionary devices of the age [...] of immense practical application not only on earth but in seeking out the wonders of the universe", und im September 1963 verhieß die Überschrift eines Artikels des New York Times Magazine: „Laser Lights Up the Future“. ${ }^{4}$ Angesichts der besonderen Eigenschaften der Laserstrahlung - enger Frequenzbereich (monochrom), scharfe Bündelung, hohe Intensität, große Kohärenzlänge - war von Anfang an klar, dass es zumindest theoretisch ein überaus breites Anwendungspotential des Lasers in der Grundlagenforschung sowie in technischen Anwendungen von der Materialbearbeitung über die Nachrichten- und Informationstechnologie, die Mess- oder die Medizintechnik bis hin zur Waffentechnik geben musste. Dank der Entdeckung immer neuer Lasermedien konnten diese hochgesteckten Erwartungen zumindest im Labormaßstab auch innerhalb weniger Jahre weitgehend bestätigt werden. Wenngleich sich die kommerzielle Nutzung der Laserstrahlung im Rahmen marktfähiger Produkte aufgrund verschiedener Faktoren noch für viele Jahre als schwierig erwies, galt der Laser von Anfang an als eine Schlüsseltechnologie für die wissenschaftliche Grundlagenforschung sowie für kommerzielle und vor allem für militärische Anwendungen. 
„I feel as do others here that the Laser may be the biggest breakthrough in the weapons area since the atomic bomb", äußerte 1962 der Chef des US-Army Ordnance Missile Command, Generalmajor August Schomburg (zit. nach Seidel 1988: 12). Nicht zu Unrecht fragte angesichts solcher Reaktionen im Februar 1963 die amerikanische Zeitschrift Reader's Digest im Hinblick auf den Laser: „Light of Hope - or Terror?" Und es scheint als sei vom „Light of Terror“ zunächst die größere Faszination ausgegangen. „,Light Ray’ - Fantastic Weapon of the Future" lautete im April 1962 die Überschrift eines Zeitungsartikels in den U.S. News, und in Reader's Digest war im Februar 1963 zu lesen: „Many experts predict that someday the laser's,bullets of light' may usher in a kind of warfare so far envisaged only in science fiction". In Deutschland stellte das Nachrichtenmagazin Der Spiegel unter der Überschrift „Waffenstrahlen - Aladins Lampe“ im Frühjahr 1962 fest:

[D]i.e. todbringenden Licht- und Hitzestrahlen, seit Jahrzehnten nurmehr Phantasiegebilde der Science-Fiction-Schreiber, sind in den Bereich seriöser wissenschaftlicher Forschung gerückt. [...]Wenn es gelängeLaser von hinreichender Größe zu bauen, so würde schließlich möglich werden, was den Militärs als perfekte Raketenabwehrwaffe vorschwebt: die Lichtkanone, unter deren Strahl Raketen und Bomber zu Staub verglühen. ${ }^{6}$

Unter Überschriften wie „Todesstrahlen“ (Umschau 1962), „Lichtkanone“ (Elektronik-Zeitung 1963) oder „Strahlen gegen Rakete“ (Militärwesen 1963) griffen andere Zeitschriften in der Bundesrepublik ${ }^{7}$ derartige Überlegungen auf. Einschlägige Fachblätter, wie Soldat und Technik oder Wehr und Wirtschaft, wiesen zwar darauf hin, dass die „Todesstrahlen noch außerhalb der technischen Möglichkeiten“ lägen und versicherten, dass „die sensationellen Berichte, die in der letzten Zeit über'LASER-Lichtstrahlen als Waffe der Zukunft' verbreitet wurden, [...] noch auf Spekulationen" beruhten (Bender 1962; Weiss 1962). Andererseits hatten in den USA bereits wenige Monate nach Maimans erstem Laser, also gut zwanzig Jahre vor den Plänen der Regierung Reagan zur Strategic Defense Initiative (SDI), die Hughes Aircraft Company und die Firma Martin Marietta damit begonnen, hauseigene Programme zur Entwicklung von Strahlenwaffen auf Laserbasis einzurichten, die u.a. auch auf die Zerstörung feindlicher Weltraumwaffen zielten (Seidel 1988: 13).

Ähnlich wie in den USA wurde auch in Deutschland die Erfindung des Lasers sowohl in der Öffentlichkeit wie auch in Kreisen von Fachleuten euphorisch gefeiert. Autoren bezeichneten den Laser als ein „Wunderwerkzeug", eine „Wunderlampe“ oder schlicht als „Aladins Lampe“, die nicht nur Tod und Verderben zu bringen vermochte, sondern auch in der Lage sein sollte, riesige Entfernungen auf einen hunderttausendstel Millimeter genau zu messen oder Raumschiffe im Weltall drahtlos mit Energie zu versorgen. ${ }^{8}$ Der deutsche Raketenforscher Eugen Sänger versprach sich 1962 vom Laser gar „eine dauernde Befriedung der Luft- und Raumfahrt“. 9 Solche 
und ähnliche Äußerungen deuteten neben aller Euphorie auf die Ambivalenz hin, mit der die Erfindung des Lasers in Fachkreisen und der Öffentlichkeit gerade zu einem Zeitpunktbetrachtet wurde, als der Kalte Krieg mit dem Bau der Berliner Mauer im August 1961 einen seiner Höhepunkte erreicht hatte.

\section{Die militärische Laserforschung in den USA}

In den USA war das Interesse des Militärs an der Laserforschung bereits 1958durch Charles Townes bei der dem Department of Defence (DoD) unterstehenden Advanced Research Projects Agency (ARPA) geweckt worden. Die ARPA war damals gerade erst zusammen mit der NASA als F\&EFörderungseinrichtung der US-Regierung gegründet worden und wandelte sich bald darauf in die zentrale Agentur des DoD zurÜberführung von Ergebnissen der Grundlagenforschung in angewandte Militärtechnologie, wobei ihr auch eine zentrale Rolle in der Förderung der US-Laserforschung zufiel. ARPA unterstützte im Laserbereich nicht nur Industrieunternehmen, sondern auch zahlreiche Universitäten durch Forschungsaufträge sowie finanzielle Zuwendungen zur Geräteanschaffung und die Durchführung von Tagungen (vgl. Bromberg 1991: 102 f.; Seidel 1987).

Neben ARPA - und teilweise in Konkurrenz dazu sowie auch untereinander - standen die selbständigen Forschungsförderungseinrichtungen der Teilstreitkräfte, wie das Office of Naval Research, das Army Office of Ordnance Research (AOOR) und das Air Force Office of Scientific Research, die ebenfalls militärisch orientierte Forschungsprogramme, wie etwa das für 23 Universitäten bestimmte 2,7-Millionen-Dollar-Programm für moderne Optik des AOOR, finanzierten (Seidel 1988: 14). Darüber hinaus verfügten die Teilstreitkräfte der USA über eigenständige Forschungslaboratorien, wie das Naval Research Laboratory oder die Air Force Cambridge Research Laboratories, die ebenfalls im nennenswerten Umfang „In-House“-Forschungen im Laserbereich betrieben. Allein die US-Armee gab zwischen 1962 und 1968 für die Entwicklung von Hochenergielasern, also für Strahlenwaffen, 8,8 Millionen Dollar aus, die etwa jeweils zur Hälfte in gleichermaßen erfolglose Eigenbeziehungsweise Fremdentwicklungen flossen.

Insgesamt soll das DoD in den USA 1960 für Forschung und Entwicklung im Laserbereich etwa eineinhalb Millionen US-Dollar, 1961 vier Millionen, 1962 zwölfMillionen und 1963 bereits zwischen 19 und 24 Millionen Dollar ausgegeben haben (Bromberg 1991: 102). Das Fachblatt Laser Focus schätzte 1970, dass der Anteil der militärisch finanzierten F\&E-Ausgaben im Laserbereich in den USA bis zum Jahr 1969 aufetwa 115 Millionen Dollar angestiegen sei und zu diesem Zeitpunkt rund 64 Prozent des gesamten Lasermarktes ausgemacht habe. ${ }^{10}$ Die weitere Prognose des Blattes lautete: 
Military-financed research will continue to expand, but at a slower pace than in the late 1960's. Now that rangefinders, illuminators and laser-guided bombs have proven themselves technically, the armed services are ready to buy them while shifting military R\&D emphasis away from such tactical systems to strategic systems $[\ldots] .{ }^{11}$

Laser Focus deutete mit seiner Aufzählung von Entfernungsmessern, Zielerfassern und lasergesteuerten Bomben an, in welchen Bereichen die militärische Laserforschung in den USA in den 1960er Jahren Erfolge aufzuweisen hatte. Es waren weniger die ambitiösen Strahlenwaffen auf der Basis von Hochenergielasern als vielmehr die eher bescheidenere Ziele verfolgenden, auf den Wegen traditioneller Forschung und Entwicklung wandelnden Programme für low-energy laser, die zu ersten militärischen Anwendungen des Lasers führten. Dennoch verfolgten die Militärs in den USA beide Forschungsrichtungen weiter. In den zwanzig Jahren zwischen 1960 und 1980 wurden in den USA schätzungsweise jeweils eineinhalb, zusammen also drei Milliarden US-Dollar für die militärische Laserforschung und -entwicklung ausgegeben. 1980 - und damit drei Jahre bevor Ronald Reagan sein SDIProgramm verkündete - gab das US-Militär 453 Millionen Dollar für Laser und Lasersysteme aus, was einem Anteil von 60 Prozent am Gesamtvolumen des Marktes entsprach, und dominierte damit auch zu diesem Zeitpunkt, trotz wachsender ziviler Märkte, noch eindeutig die Laserindustrie der USA (Seidel 1988: 18).

In der Frühzeit der Laserentwicklung Ende der 1950er Jahre war zumindest in den USA die Dominanz des Militärs nahezu umfassend, da sich ein ziviler Lasermarkt im nennenswerten Umfang erst in der zweiten Hälfte der 1960er Jahre zu entwickeln begann. Das Militär trat dabei sowohl als Hauptsponsor der Forschung alsauch als Hauptabnehmer der entwickelten Produkte auf. Da sich bald herausstellte, dass im Laserbereich aufgrund zahlreicher technischer Probleme praktische kommerzielle Anwendungen nur auf lange Sicht zu erreichen und deshalb nur schwer zu finanzieren waren, wechselten zudem viele Universitäts- und Industrieforscher zu solchen Forschungen und Entwicklungen, für die von militärischer Seite Geld zur Verfügung stand oder Geld zu akquirieren war (Seidel 1988: 14). ${ }^{12}$ Die Folgen einer solchen Entwicklung hin $\mathrm{zu}$ einer immer engeren Verbindung von Wissenschaft, Industrie und Militär zeichneten sich in den USA bereits seit den 1950er Jahren in der Entstehung eines gewaltigen wissenschaftlich-industriell-militärischen Komplexes ab, in dem einerseits zahllose Wissenschaftler Arbeit und Freiräume für ihre Forschungen fanden, in dem andererseits aber auch die Rolle dieser Wissenschaftler sowie die ihrer Arbeit und ihrer Wissenschaft, einschließlich der vermeintlich zweckfreien Grundlagenforschung, völlig neu definiert wurde (Mendelsohn et al. 1988). Paul Forman formulierte im Hinblick auf diesen Zusammenhang in seiner grundlegenden Studie Behind 
Quantum Electronics: National Security as Basis for Physical Research in the UnitedStates, 1940-1960 aus dem Jahr 1987 die These

that here American physics, accelerating its historic quantitative growth, underwent a qualitative change in its purposes and character, an enlistment and integration of the bulk of its practitioners and its practice in the nation's pursuit of security through ever more advanced military technologies (Forman 1987: 150).

Dass der Laser in dieser Entwicklung nur das vorläufig letzte Glied in einer ganzen Kette von Ereignissen darstellte, wird aus einer Feststellung von General Arthur Trudeau, dem Chef der Forschungs- und Entwicklungsabteilung in der US-Armee, aus dem Jahre 1962 deutlich:

Basic research, being but the applied research of tomorrow, is the key to technological progress. [...] Army Research and Development is maximising the great sientific discoveries of today and continually pressing for more progress in all fields. $[\ldots]$ Electronics $[\ldots]$ has seen a quantum jump in the last 10 years. Basic research already has led to the microminiaturization of electronic components. [...] Other marvels in the electronic field are the ruby maser [...] And now, the LASER and IRASER are in sight (Trudeau 1962; zit. nach Forman 1987: 151).

Nach Ansicht Robert Seidels verstärkte der Laser noch das Interesse der Militärs an der Grundlagenforschung, und dieses verstärkte Interesse wirkte wiederum auf die amerikanische Laserforschung zurück, indem es gleichzeitig den Charakter dieser Forschung von der Small Science zur BigScience, von offener zu klassifizierter Forschung und von der akademischen Forschung zur eigenen Forschungin vertraglich gebundenen Laboratorien veränderte.

Lassen sich aber nun diese Forschungsergebnisse von Forman, Bromberg oder Seidel für die Laserforschung in den USA auch auf deren wichtigsten europäischen Verbündeten und NATO-Partner, die Bundesrepublik Deutschland übertragen? In der Bundesrepublik setzten die ersten Forschungen zum Laser unmittelbar nach Bekanntwerden der erfolgreichen US-amerikanischen Experimente um die Jahreswende 1960/1961 ein. ${ }^{13}$ Die ersten Laser in den Forschungslaboratorien von Industrie und Hochschulen konnten bereits Anfang beziehungsweise im Laufedes Jahres 1961 realisiert werden. Frühe Zentren bundesdeutscher Laserforschung waren dabei die Technischen Hochschulen Berlin, Braunschweig, Karlsruhe, München und Stuttgart sowie die Unternehmen Zeiss in Oberkochen, Siemens in München oder auch die kleine Firma Impulsphysik in Hamburg. Neben dem Interesse an Forschung und Entwicklung im Bereich der wissenschaftlichen Grundlagen und praktischen Anwendungen des Lasers im zivilen Bereich spielten dabei auch in Deutschland von Anfang an militärische Überlegungen eine Rolle. Für deren Analyse gilt es zunächst einen Blick auf die Rahmenbedingungen der bundesdeutschen militärischen Forschungsförderung zu werfen, bevor deren Rolle für die Entwicklung von Laserforschung und Lasertechnik in der Bundesrepublik Deutschland in den 1960er Jahren im Detail analysiert werden kann. 


\section{Die Forschungsförderung durch das Bundesministerium für Verteidigung in den 1960er Jahren}

Nach Angaben der Zeitschrift Wehr und Wirtschaft aus dem Jahre 1965 betrugen die Ausgaben des amerikanischen Verteidigungshaushaltes für Forschung im Jahre 1963 etwa 7,5 Milliarden US-Dollar oder 13,7 Prozent desgesamten Verteidigungshaushalts. In Großbritannien lag der gleiche Anteil bei rund 14 Prozent oder umgerechnet 2,8 Milliarden DM, während der Forschungsanteil des Haushalts des Bundesministers für Verteidigung (BMVg) zur gleichen Zeit lediglich 3,03 Prozent beziehungsweise 547,8 Millionen DM betrug. ${ }^{14}$ Bei diesem Betrag handelte es sich allerdings um die Ausgaben für Verteidigungsforschung und wehrtechnische Entwicklung zusammen, von denen auf Forschungsaufgaben im engeren Sinne - einschließlich zweckgerichteter Grundlagenforschung und angewandter Forschung - lediglich 10,3 Prozent oder 56,3 Millionen DM entfielen (vgl. Tabelle 1). ${ }^{15}$ Bis zum Ende des Jahrzehnts erhöhten sich die Ausgaben für Forschung und Entwicklung im Verteidigungshaushalt auf 1,15 Milliarden DM (5,9 Prozent des Verteidigungshaushalts), von denen jetzt 159,3 Millionen DM (13,8 Prozent) für Forschung im engeren Sinne ausgegeben wurden. ${ }^{16}$ Erscheinen diese Beträge gegenüber den Forschungsaufwendungen etwa des US-Militärs auch als sehr gering, so erhalten sie im bundesdeutschen Vergleich etwa mit den zur gleichen Zeit von der DFG im Rahmen ihrer Schwerpunktprogramme für die Forschungsförderung ausgegebenen Mittel (1963: 33,95 Mio. DM beziehungsweise 1969: 56,94 Mio. $\mathrm{DM}^{17}$ ) eine gänzlich andere Dimension.

Tabelle 1 Entwicklung des Etats und der F\&E-Ausgaben des Bundesministeriums für Verteidigung 1959-1970

\begin{tabular}{llllll}
\hline Jahr & Etat insgesamt (in Mrd. DM) & \multicolumn{4}{l}{ davon F\&E-Ausgaben (in Mio. DM) } \\
\cline { 3 - 5 } & & insges & Prozent & Forschung im eng. & Sinne \\
\hline 1959 & 8,5 & 155,8 & 1,83 & \\
$1960 *$ & 7,4 & 183,0 & 2,47 & \\
1961 & 11,6 & 381,3 & 3,28 & \\
1962 & 15,5 & 410,3 & 2,65 & & \\
1963 & 18,1 & 547,8 & 3,03 & 56,3 & 10,3 \\
1964 & 17,5 & 649,2 & 3,71 & & \\
1965 & 17,8 & 704,2 & 3,96 & & \\
1966 & 18,0 & 760,6 & 4,23 & & \\
1967 & 19,7 & 968,9 & 4,92 & & 14,7 \\
1968 & 17,3 & 985,3 & 5,69 & 13,8 \\
1969 & 19,1 & 1060,4 & 5,55 & 157,6 & 159,3 \\
1970 & 19,4 & 1152,3 & 5,94 & 159 \\
\hline
\end{tabular}

* Rumpfrechnungsjahr vom 1.4. bis 31.12.1960 
Von den rund 160 Millionen DM des BMVg für Forschungsmittel im engeren Sinne flossen nach Angaben des Forschungsberichts (IV) der Bundesregierung etwa 65 Millionen über Forschungsverträge an einzelne Forscher, wissenschaftliche Einrichtungen und die Industrie, während rund neunzig Millionen DM zur Grundfinanzierung einer Reihe selbständiger Forschungseinrichtungen ausgegeben wurden, die in enger Verbindung zum Verteidigungsministerium standen. ${ }^{19}$ Im Einzelnen handelte es sich dabei um die Deutsche Forschungs- und Versuchsanstalt für Luft und Raumfahrt e.V. (DFVLR) und die Fraunhofer-Gesellschaft für angewandte Forschung e.V. (FhG), deren Etats im Umfang von bis zu zwanzig Prozent durch das BMVg finanziert wurden, sowie die Institute der Gesellschaft zur Förderung der Astrophysikalischen Forschung e.V.beziehungsweise der Forschungsgesellschaft für Angewandte Naturwissenschaften e.V. (FGAN) in Werthoven bei Bonn, Tübingen und Karlsruhe, die gänzlich aus demEtat des BMVg versorgt wurden (Gugel u. a. 1986: 40). Darüber hinaus unterhielt das BMVg als eigene Forschungseinrichtungen die Forschungsanstalt der Bundeswehr für Wasserschall- und Geophysik (FWG) in Kiel sowie seit 1959 das gemeinsam mit Frankreich betriebene Deutsch-Französische Forschungsinstitut St.-Louis (ISL), das sich auf dem Gebiet der Ballistik und des Waffenwesens betätigte. Im Rahmen dieser vom BMVg geförderten Institutionen kam der Fraunhofer-Gesellschaft eine besondere Bedeutung zu, da die FhG sowohl die Verwaltungshilfe bei der Abwicklung von Forschungsaufgaben des Verteidigungsministeriums an den Hochschulen übernahm als auch bis 1965 fünf Institute errichtete, die Forschungsaufgaben im Sinne des BMVg erledigten. ${ }^{20}$ Diese Regelung bedeutete - bewusst im Gegensatz zum amerikanischen Vorbild - ein Votum gegen die Schaffung einer größeren Zahl eigener Forschungsanstalten für den militärischen Bereich in der Bundesrepublik und bot zudem die Möglichkeit, für die militärische Forschung Institute zu schaffen, die einerseits nicht den Offenlegungsnormen der Hochschulen, andererseits aber auch nicht der direkten militärischen Befehlsgewalt unterworfen waren (Stamm 1981: 253 f.).

Wichtig für die militärische Forschungsförderung in der Bundesrepublik war schließlich noch die internationale wissenschaftlich-technische Zusammenarbeit im militärischen Bereich vor allem mit den NATO-Partnern, die unter der Leitung der direkt dem Nordatlantik Ratunterstehenden Conference of National Armament Directors durch die Defense Research Group (DRG) koordiniert wurde (Schall 1969). Eng verbunden mit der DRG war der Wissenschaftsausschuss (Science Committee) der NATO, in dem alle Fragen der Verteidigungstechnik beraten wurden, die eine längere wissenschaftliche Bearbeitung erforderten. Der Wissenschaftsausschuss stellte durch die von ihm regelmäßig veranstalteten NATO-Sommerschulen darüber hinaus den internationalen wissenschaftlichen Informationsaustausch zwischen Fachleuten der Rüstungsindustrie und ausgesuchten Studenten und 
Wissenschaftlern aus dem akademischen Bereich sicher. Eine Reihe bilateraler Abkommen der Bundesrepublik mit NATO-Partnern, wie den USA, Kanada, Großbritannien, den Niederlanden oder auch Frankreich, sicherte zudem die Durchführung gemeinsamer militärischer Forschungsarbeiten sowie den gegenseitigen Austausch von militärisch relevanten Forschungsergebnissen. ${ }^{21}$ Neben DRG und Wissenschaftsausschuss verfügte die NATO auch über einige eigene Forschungsinstitutionen, wie dem mit Unternehmensforschung, Systemanalyse und Nachrichtenwesen befassten SHAPE Technical Centre (STC) in Den Haag, dem mit Fragen der U-Bootortung beschäftigten SACLANT Anti-Submarine Warfare Centre (SACLANTCEN) bei La Spezia in Italien sowie der in der Luft- und Raumfahrtforschung aktiven Advisory Group for Aerospace Research and Development (AGARD) in Paris.

\section{Die Förderung der Laserforschung und Lasertechnik durch das Bundesministerium für Verteidigung}

Sowohl die NATO-Kontakte und -Einrichtungen wie auch die mit dem BMVg eng zusammenarbeitenden Forschungsinstitutionen des Inlandes spielten bei der Erforschung und Entwicklung einer militärisch nutzbaren Lasertechnologie in der Bundesrepublik eine Rolle. Erstmals öffentlich erwähnt wurden derartige Forschungen im Bundesforschungsbericht II 1967. Im Abschnitt zur Forschungsförderung des BMVg war dort unter dem Förderschwerpunkt „Informationsgewinnung" der Verteidigungsforschung und -entwicklung zu lesen:

Die Laser-Technik konnte bei der Entwicklung von Geräten zur Messung von Entfernungen nutzbar gemacht werden. Die Anwendung dieser Technik verspricht eine erhebliche Verbesserung der Feuerleitung. ${ }^{22}$

Dieser knappe Hinweis deutet bereits an, dass sich in der Bundesrepublik in den 1960er Jahren die militärische Förderung der Laserforschung und Lasertechnik mit der Entwicklung von Laserentfernungsmessern auf einen Bereich konzentrierte, der schnelle Erfolge bei nicht zu großem Entwicklungsaufwand versprach. Bereits Ende der 1950er Jahre war allgemein bekannt, dass sich im Falle einer Weiterentwicklung des Mikrowellenradars zum optischen Radar die Genauigkeit, mit der sich ein Objekt würde lokalisieren und von einem anderen Objekt würde unterscheiden lassen, erheblich steigern ließe. Darüber hinaus würden durch die erheblich kürzeren Wellenlängen die Antennen für optisches Radar nur einen Bruchteil der Größe des Mikrowellenradars haben, was sie vor allem für den Einbau in mobilen Systemen wie Panzern, Flugzeugen, Satelliten oder gar tragbaren Geräten für Infanteristen geeignet erscheinen ließ. Derartige optische Radarsysteme waren 
zudem bereits im Versuchsstadium vorhanden, wenn auch das für ihren Betrieb notwendige kohärente Licht noch auf komplizierten Wegen erst aus herkömmlichem Licht erzeugt werden musste. Die dabei auftretenden Probleme geringer Leistung, mangelhafter Monochromasie und schlechter Bündelung des Lichtes, die einen Einsatz solcher Geräte nur auf kurze Entfernung und in Abwesenheit von Sonnenlicht ermöglichten, würden durch das Laserlicht schlagartig beseitigt werden. Gleiches galt für die bislang in Panzern oder auf Schiffen verwendeten herkömmlichen optischen Basis-Entfernungsmessgeräte, deren Reichweite und Genauigkeit sich bei Verwendung des Lasers, bei gleichzeitiger Reduzierung der Baugröße, erheblich steigern ließ.

In den USA war die Realisierbarkeit von Laser-Entfernungsmessern durch zwei, von der Air Force beziehungsweise der U.S. Army geförderte Arbeitsgruppen der Hughes Aircraft Company bereits Ende Januar 1961 grundsätzlich nachgewiesen worden (vgl. Bromberg 1991: 126 f.). Im Frühjahr 1961 gelang in den Forschungslaboratorienvon Hughes in Malibu/Kalifornien mit der Entwicklung der Q-Switch-Technikein weiterer wichtiger Schritt auf dem Weg zum Bau eines wirklich funktionsfähigen Laser-Radars beziehungsweise Laserentfernungsmessers, da mit Güteschaltern (Q-Switch) ausgestattete Laser aufgrund ihrer leistungsstarken Lichtimpulse besonders gut für die militärische Entfernungsmessung geeignet waren. Über die Methode des Güteschalters sowie dieMöglichkeiten und den Stand der militärischen Laserentwicklung konnten sich Experten des Bundesverteidigungsministerium in Bonn offenbar erstmals ausführlich auf der vom 2. bis 4. April 1962 in Den Haag veranstalteten NATO-Laser-Konferenz unterrichten, an der Vertreter der BMVg-Fachreferate Wehrforschung (T II) sowie Fernmeldetechnik, Elektronik, Optik und Akustik (T VI) teilnahmen. ${ }^{23}$ Auf dieser Tagung wurden in 24 Vorträgen die wesentlichen Grundlagen und Anwendungen des Lasers behandelt, wobei die Entfernungsmessverfahren im Vordergrund standen. ${ }^{24}$ Entsprechende Versuche waren zu diesem Zeitpunkt offenbar nicht nur in den USA, sondern auch in Frankreich bereits im Gange. $\mathrm{Zu}$ den vorläufigen Ergebnissen dieser Versuche berichteten die BMVgVertreter:

Über die Frage der Rückstrahlung der Atmosphäre bei schlechten Wetterbedingungen konnten noch keine Angaben gemacht werden. Zunächst ist eine solche Anlage als Schönwettergerät anzusehen. ${ }^{25}$

Als weitere Anwendungen des Lasers im militärischen Bereich, „die aber noch sehr in der Zukunft" lägen,wurden auf der Haager Konferenz Nachrichtenverbindungen „durch ausgepumpte Röhren mit Kanalzahlen von mehreren 100 Millionen“, Aufklärungsverfahren mit aktivem line scan, optisches Radar und die Feuerleitung bei Lenkwaffen genannt. Strahlenwaffen auf der Basis von Hochenergielasern, wie sie in den USA bereits erforscht wurden, waren dagegen offensichtlich kein Thema der Tagung. Aus den Vorträgen und 
Diskussionen der NATO-Laser-Konferenz zogen die beiden Vertreter der Hardthöhe für die weitere Förderung der militärischen Laserforschung in der Bundesrepublik die Schlussfolgerung:

Die bei T II begonnenen Forschungsarbeiten über Züchtung von Laserkristallen, Anpassung der Erregungslichtquellen, Verbesserung der "Güte" des Lasers sowie der Modulationsmöglichkeiten sollten fortgesetzt werden. Daneben sollte von T VI $6^{26}$ die Erstellung eines „Versuchsmodells“ eines E-Messers veranlaßt werden, um Erkenntnisse zu sammeln, die für eine kommende internationale Entwicklung auf diesem Gebiet unbedingt erforderlich sind. Zweckmäßig würde hierbei die Firma Früngel eingeschaltet, die durch vorausgehende Forschungsverträge bereits Untersuchungen auf dem E-Meß-Gebiet durchgeführt hat. Eine enge Zusammenarbeit T II $3^{27}$ und T VI 6 wäre hierbei erforderlich, so dass jeweils die Fortschritte der Forschungsarbeiten in das Versuchsmodell eingearbeitet werden könnten. ${ }^{28}$

Wie aus diesen Schlussfolgerungen hervorgeht, waren erste Untersuchungen zur Lasertechnik vom BMVg zu dieser Zeit bereits eingeleitet worden. Das für die optische Forschung zuständige Fachreferat der Abteilung für Wehrforschung im BMVg hatte in dieser Frage schon Anfang Juli 1961 mit der Firma Optische Werke Ernst Leitz GmbH Wetzlar Kontakt aufgenommen und erhielt daraufhin von dem Unternehmen einen Bericht über „Forschungsu. Anwendungsmöglichkeiten auf dem Gebiet der optischen Maser (Laser) im Rahmen der FA. E. Leitz GmbH“ zugesandt, der, so das Begleitschreiben, „lediglich als Anhaltspunkt für eine mögliche Entwicklung“ gedacht war und zunächst nur die Aufgabe hatte, zu klären, „ob von Ihrer Seite Interesse an einer derartigen Sache“ bestünde. ${ }^{29}$ Obwohl der Leitz-Bericht eine bald realisierbare Anwendung des Lasers lediglich ,in Form eines mit dem Dopplereffekt arbeitenden Geschwindigkeitsmeßgerätes“ sah und ansonsten unter Hinweis darauf, dass „die gesamte Lasertechnik noch in den Kinderschuhen“ steckte, über weitere Anwendungsmöglichkeiten nicht spekulieren wollte, reagierte das BMVg umgehend. Zur Klärung weiterer Schritte lud das Ministerium Vertreter der Firmen Leitz und Telefunken nach Bonn ein und schon im November konnte Leitz dem Ministerium einen ersten „Entwurf für einen Forschungsauftrag auf dem Gebiet der quantenmechanischen lichtoptischen Verstärker und Oszillatoren (Laser)“ "vorlegen. ${ }^{30}$

Dieser Entwurf sah vor, dass die Firma Leitz im Auftrag des BMVg sowohl Festkörper- alsauch Helium-Neon-Gaslaser bauen und auf ihre Strahlungseigenschaften untersuchen sollte. Die für den Festkörperlaser notwendigen Kristalle wollte Leitz auf dem freien Markt besorgen und dann selbst weiter bearbeiten. Darüber hinaus bot die Firma an, in einem gegebenenfalls abzuschließenden weiteren Forschungsvorhaben die Züchtung von neuen Laser-Kristallen selbst zu übernehmen. Der Bau des Helium-Neon-Laser war nach dem Entwurf in zwei Ausführungen geplant - einmal mit planen und einmal mit sphärischen Endflächen -, um die entsprechenden Justierungsprobleme erforschen zu können. Sowohl für den Festkörperlaser 
als auch für den Gaslaser sollte schließlich noch „die Anpassung an nachgeschaltete optische Glieder [...] (Optische Antennen)“ untersucht werden. Für die beim Bau der Laser anfallenden „reinen elektrischen Aufgaben" war eine enge Zusammenarbeit mit der Firma Telefunken in Ulm vorgesehen. Nachdem Ende November 1961 in einer gemeinsamen Besprechung in Ulm die Fragen der Kooperation zwischen Leitz und Telefunken geklärt waren, ${ }^{31}$ kam es auf der Grundlage des „Entwurfs“ im März 1962 zum Abschluss eines Forschungsvertrags zwischen dem BMVg und Leitz zum Thema „Forschungsarbeiten über quanten-mechanische, lichtoptische Verstärker und Oszillatoren (Laser)“. Er sah eine Laufzeit von 24 Monaten vor und wurde von der Hardthöhe, entsprechend einer Vorkalkulation der Firma Leitz, mit rund 420.000 DM vergütet. ${ }^{32}$ Der für Ende 1963 vorgesehene Abschluss der Arbeiten kam allerdings nicht zustande. Die Firma Leitz sah sich gezwungen, noch vor Ablauf des Vertrags eine einjährige Verlängerung zu beantragen. Schlussendlich lag ihr Abschlussbericht zum Forschungsvorhaben in Bonn sogar erst im November 1967 vor. $^{33}$

Bereits 1962 hatte sich das BMVg entschlossen, mit der Firma Leitz den bereits im ersten Forschungsvertrag angeregten, zusätzlichen Forschungsauftrag über Arbeiten zur Züchtung von Laser-Kristallen im Umfang von rund 280.000 DM und einer Laufzeit von 15 Monaten abzuschließen. Die Begründung für diesen Forschungsvertrag lautete:

Es wird für unbedingt erforderlich gehalten, dass auch in der Bundesrepublik in gewissem Umfang auf dem Gebiet der Züchtung von Laser-Kristallen gearbeitet wird. Die Gründe dafür liegen einmal in der Bedeutung des Lasers selbst und zum zweiten in der Schwierigkeit, trotz großen allgemeinen Angebotes qualitativ hochwertige Kristalle zu beziehen. ${ }^{34}$

Nachdem sich bei den Arbeiten zum ersten Vertrag ergeben hatte, dass die angekauften, teilweise extrem teuren Laserkristalle ${ }^{35}$ keineswegs immer den in sie gesetzten Erwartungen entsprachen, sollte es das Ziel der Arbeiten bei Leitz sein, sowohl neue Züchtungsverfahren für spannungsfreie Rubin-Kristalle zu erkunden, um dadurch homogenere Kristalle mit einer besseren Bündelungsschärfe des Strahles zu erreichen, als auch Alkalihalogenid-Kristalle nach dem Absenkverfahren mit geeigneten Dotierungsstoffen, wie Seltene Erden, Uran oder Chrom, herzustellen. Im Rahmen des Vertrags führte Leitz schließlich 166 Kristallzüchtungen durch, wobei sich von 150 gezüchteten Calciumfluorid-Kristallen nur 84 zur weiteren Bearbeitung geeignet erwiesen. Von diesen 84 Kristallen konnten anschließend nur 31 Stäbe zur Messung gebracht werden, wobei lediglich vier (!) Prüfstäbe, einer davon sogar noch zweifelhaft, Lasertätigkeit zeigten. ${ }^{36}$ Um überhaupt diese Ergebnisse erzielen zu können, musste die Vertragslaufzeit um ein Jahr bis Ende 1964 verlängert werden. Der endgültige Abschlussbericht der Firma lag schließlich erst im Dezember 1965 vor. 
Die insgesamt bescheidenen Ergebnisse der im Auftrag des BMVg bei Leitz und Telefunken durchgeführten Grundlagenforschungen zeigen, dass man in der Bundesrepublik 1962 zumindest in der militärischen Laserforschung noch ganz am Anfang stand. Angesichts der auf der Tagung der NATO-Expertengruppe für optische Instrumente im Dezember 1962 in Paris vorgestellten „erstaunlich vielfältigen“ Arbeiten auf diesem Gebiet in den USA und Großbritannien kam der Vertreter der Hardthöhe auf dieser Tagung zu der Erkenntnis:

Einige der Entwicklungen sind so weit fortgeschritten, dass mit Einführung z.B. der Laser-Technik zur Entfernungsmessung im Kampfwagen etwa ab 1965 zumindest in den USA gerechnet werden kann. [...] Auf allen diesen Gebieten laufen in der BRD keine nennenswerten Entwicklungen, so dass wir völlig auf die Informationen aus dem Ausland angewiesen sind. ${ }^{37}$

Die Tagung der Pariser Expertengruppe im Dezember 1962 bestätigte damit erneut, was bereits auf der Den Haager Laserkonferenz im April in Ansätzen deutlich geworden war: Die NATO-Partner USA, Großbritannien und Frankreich begnügten sich längst schon nicht mehr mit Grundlagenforschungen zum Laser, sondern arbeiteten bereits ernsthaft an praktischen Laseranwendungen für ihre Streitkräfte, wobei der Laserentfernungsmessung ganz offensichtlich die größten Chancen auf eine baldige Realisierung eingeräumt wurden. Dass die Bundeswehr dabei nicht abseits stehen wollte, ergibt sich aus einer Stellungnahme des Fachreferats für Infrarot- und Lichttechnik der Abteilung für Wehrtechnik am BMVg vom Juli 1963:

Der Wunsch der Truppe geht dahin, den bisher in Panzern verwendeten optischen Entfernungsmesser durch einen Laser-Entfernungsmesser zu ersetzen. Die neue Laser-Technik gibt die Möglichkeit der Entfernungsmessung durch Messung der Laufzeit des Lichtimpulses. Der Vorteil gegenüber der bisher verwendeten Methode der optischen Winkelmessung besteht in der von der Entfernung unabhängigen Meßgenauigkeit, dem geringeren Gewicht, den geringeren Abmessungen und somit der leichteren Einbaumöglichkeit in Panzern. ${ }^{38}$

Unmittelbar nach der Haager NATO-Konferenz begann denn auch das BMVg mit ersten Überlegungen zur Entwicklung eigener bundesdeutscher Laserentfernungsmessgeräte. Als Partner bot sich dafür die Firma Impulsphysik Dr.-Ing. Frank Früngel GmbHin Hamburg-Rissen an, die im Auftrag der Hardthöhe bereits seit 1958 unter dem Projektnamen „Nebelauge II“ an der Verbesserung der herkömmlichen optischen Verfahren zur Entfernungsmessung arbeitete. ${ }^{39}$ Der Inhaber der Firma, Frank Früngel, hatte als einer der beiden bundesdeutschen Referenten auf der NATO-Laser-Tagung darüber berichtet, dass es seiner Firma gelungen sei, „,repetierende Blitze eines Laser-Kristalles mittels zweier Pumplichtquellen bezw. zweier elektrischer Stromkreise zu erzeugen". ${ }^{40}$ Früngel sowie weitere Mitarbeiter seiner Firma beschäftigten sich außerdem mit dem Einsatz von Lasern in der Kurzzeitphotographie und schienen damit durchaus kompetente Partner für das 
BMVg zu sein (Früngel 1962; Früngel 1963a, b; Ebeling 1963). Ende April 1962, also im unmittelbaren Anschluss an die NATO-Laser-Konferenz in Den Haag, wandte Früngel sich an die Abteilung Wehrforschung des BMVg mit dem Vorschlag,

die LASER-Technik mit der im Rahmen von „Nebelauge II“ entwickelten Bildwandlertechnik bezw. Kerrzellen-Bildwandler-Technik so zu kombinieren, dass anstelle des unmittelbaren Funkenblitzes der scharf gerichtete LASER-Blitz genutzt wird. Es sollen etwa 25 Blitze hintereinander zur Aussendung kommen, so dass der Bildwandler ein auch visuell auswertbares Bild liefert. Nur das menschliche Auge als Kriterium ist in der Lage, aus der Aufhellung des Zielobjektes auf die richtige Laufzeit des Beleuchtungsimpulses zu schließen. ${ }^{41}$

Den Vorteil des von ihm angeboten Systems sah Früngel vor allem darin, dass es im Gegensatz zu den in Frankreich realisierten Geräten, nicht nur bei klarer Sicht einsetzbar sein sollte, sondern darüber hinaus auch eine direkte visuelle Identifizierung des Zielobjekts erlauben würde. Der zur Realisierung dieser Vorstellungen von Früngel angeregte Vertrag mit einer Vertragssumme von 120.000 DM zum Thema „Untersuchungen zur Einbeziehung von LaserStrahlungsquellen in die in den vorhergehenden Untersuchungen erarbeiteten Entfernungsmeßverfahren mit Bildwandler- bzw. Kerrzellenverschluß" kam noch 1962 unter der Kurzbezeichnung „Nebelauge III“ zustande. ${ }^{42}$ Vereinbart wurde, die Untersuchungen soweit durchzuführen, wie „es zur Demonstration der Möglichkeit eines derartigen Verfahrens notwendig“ sei.

Das BMVg faszinierte an dem von Früngel vorgeschlagenen Verfahren vor allem die geplante Ergänzung der für Laser-Entfernungsmesser üblichen Methode der Impulslaufzeitmessung ${ }^{43}$ durch das bildmäßige Verfahren zur eindeutigen Identifizierung des angemessenen Objekts, hätte dies doch eine fast revolutionäre Erweiterung der inzwischen bekannt gewordenen amerikanischen oder französischen Versuche zur Laserentfernungsmessung bedeutet. Gerade diese Erweiterung sollte aber Probleme bringen und das Projekt schließlich scheitern lassen. Der Vertrag mit der Firma Impulsphysik in Hamburg zum „Nebelauge III“ musste insgesamt zweimal bis zum 30. Juni 1963 verlängert werden, bevor endlich ein Schlussbericht vorlag. ${ }^{44}$ Auch dann waren die Ergebnisse noch mehr als dürftig, da es weder gelang, die für die bildmäßige Identifikation des Zielobjekts notwendige Pulsfolge des Lasers von $25 \mathrm{~Hz}$ zu erreichen, noch aussagekräftige praktische Versuche über die Reichweite und Messgenauigkeit der Versuchsanordnung durchzuführen, da der als Herzstück des Versuchsaufbaus notwendige Bildwandler nicht zur Verfügung stand. ${ }^{45}$ Stattdessen angestellte Versuche mit einem Multiplier ergaben „bei hellem Sonnenlicht“ Reichweiten von einem Kilometer, die theoretisch „Entfernungen von vielleicht 2-3 km mittels eines einstufigen Bildwandlers bei $20 \mathrm{~Hz}$-Betrieb" ermöglichen sollten. Das Fazit des Berichts lautete: „Plausibel dürfte nach jetzigem Ermessen eine Entfernungsmessung in 1-3 km Distanz bei guten Bedingungen sein". ${ }^{46}$ Letztendlich ergab sich damit, 
dass die herkömmliche, „nicht-bildmäßige-Messung“ mittels Fotozelle oder Multiplier, wie sie in den USA oder Frankreich getestet wurde, sich dem von Impulsphysik vorgeschlagenen Verfahren der „bildmäßigen Messung“ als überlegen erwies. Mit anderen Worten: Das BMVg hatte erneut 120.000 DM auf denkbar schlechte Art und Weise investiert und damit seine Negativbilanz im Bereich der Förderung der Laserforschung und Lasertechnik auf 820.000 DM erhöht.

Aussagen über Umfang und Erfolg der weiteren Förderung von Laserforschung und Lasertechnik durch das BMVg in den 1960er Jahren lassen sich aufgrund der problematischen Quellenlage nur bedingt machen. ${ }^{47}$ Die zugänglichen Unterlagen ermöglichen allerdings zumindest einen ungefähren Eindruck der Förderungsbreite, ihrer inhaltlichen Schwerpunkte sowie des Informations- und Institutionsnetzwerks auf dem die militärische Laserforschung der Bundesrepublik basierte.

Schon 1962 bestanden neben den erwähnten vertraglich geregelten Beziehungen zu den Firmen Leitz und Impulsphysik nach Angaben des BMVg „mit den in der BRD auf dem LASER-Gebiet arbeitenden Firmen (Telefunken, Osram, Siemens und Zeiss) [...] eine enge Zusammenarbeit ohne finanzielle Beteiligung". ${ }^{8}$ Die genannten Unternehmen besaßen seit Jahren intensive Kontakte zum Verteidigungsministerium und gehörten $\mathrm{zu}$ den wichtigsten inländischen Ausrüstern der Bundeswehr mit elektronischen, optischen und nachrichtentechnischen Einrichtungen. In den folgendenJahre profitierten einige dieser Unternehmen ebenfalls von der direkten Forschungsförderung des BMVg im Laserbereich, wie sich etwa am Beispiel der Firma Telefunken zeigen lässt. Das Forschungsinstitut der Telefunken AG in Ulm, die als Subunternehmer ja bereits seit 1962 über den Forschungsvertrag der Firma Leitz mit Entwicklungsarbeiten zur Elektrik der Leitz-Laser beauftragt war, beschäftigte sich so 1966 im Auftrag des BMVg mit Fragen der Lichtausbreitung von Laserstrahlen unter Wasser. ${ }^{49}$ Ein Jahr später arbeitete das Unternehmen offensichtlich an einem Studienvertrag für Forschungen zum Schutz von Beobachtern gegen Laserlicht durch ein Bildwandlersystem. ${ }^{50}$ Am Ende des hier untersuchten Zeitraumes, im August 1970, gelang der nunmehrigen AEG-Telefunken AG schließlich der Abschluss eines Studienvertrages mit dem BMVg über den Einsatz von Halbleiterlasern als gepulste Strahlungsquelle für die Verwendung in Nachtsichtsystemen mit getasteten Bildempfängern, der bei einer anderthalbjährigen Laufzeit ein Volumen von 700.000 DM besaß. ${ }^{51}$ Auch die Firma Carl Zeiss in Oberkochen, deren Aktivitäten im Bereich der militärischen Laserforschungen im Folgenden noch etwas detaillierter dargestellt werden sollen, erarbeitete Ende der 1960er Jahre für das BMVg eine Studie über „Laser-Schutzfilter im sichtbaren Spektralbereich“.52 Für Laserarbeiten der Firma Siemens ließen sich in den Akten des BMVg zwar für die 1960er Jahre keine direkten Nachweise finden, doch berichteten ehemalige Mitarbeiter der Laserarbeitsgruppen des 
Unternehmens, dass ein nicht unerheblicher Teil ihrer Arbeiten zu dieser Zeit aus Mitteln der Hardthöhe finanziert wurden. ${ }^{53}$ Ende der 1970er Jahre wurde in der Bundeswehr als erster Laserentfernungsmesser jedenfalls der von Siemens entwickelte LEM 3/1 im Flak-Panzer Gepard zur Unterstützung des Feuerleitradars eingeführt. ${ }^{54}$

Neben den bisher genannten Unternehmen lassen sich in den 1960er Jahren einige weitere vom BMVg mit Verträgen zur Laserforschung geförderte oder doch zumindest in dieser Frage in Kontakt stehende Firmen nachweisen. So hielten beispielsweise die Atlas-Werke AG Bremen im Dezember 1963 den Zeitpunkt für gekommen, das BMVg darauf aufmerksam zu machen, „dass an einen erfolgversprechenden Einsatz eines Lichtradars in Seewasser für militärische Aufgaben zu denken" sei und überreichten zur Untermauerung dieser These dem Ministerium einen Bericht mit dem Titel „Laser. Reichweiten in Seewasser", den ihre Forschungsgruppe auf der Grundlage eines intensiven Literaturstudiums der "freien und klassifizierten Literatur" erarbeitet hatte. ${ }^{55}$ Ein anderer Partner des BMVg war die Heidelberger Gesellschaft für Strahlentechnik Eltro GmbH \& Co., die 1964 an einer Studie über „Ausbreitungsgesetze Laserstrahlung“ beteiligt war und von 1964 bis 1967 im Auftrag des Ministeriums eine Studie zum „Laser-Strahlenschutz“ für rund 134.000 DM anfertigte. ${ }^{56}$ Eltro war im eigentlichen Sinne kein selbständiges deutsches Unternehmen, sondern der deutsche Vertreter des amerikanischen Laserproduzenten Hughes Aircraft Company, deren Laser sie in Lizenz baute und vertrieb. In den späten 1970er Jahren entwickelte sich Eltro zu einem der Hauptausrüster der Bundeswehr mit Laser-Entfernungsmessern, die u.a. im Kampfpanzer Leopard II oder im Artilleriebeobachtungspanzer M 113 zum Einsatz kamen. ${ }^{57}$ Auch das klassische Rüstungsunternehmen Messerschmidt-Bölkow-Blohm (MBB) in München ${ }^{58}$ beschäftigte sich Ende der 1960er beziehungsweise Anfang der 1970er Jahre mit militärischen Laserforschungen, die im Anschluss an das amerikanische Hochenergielaser-Programm allerdings die Wirkung des Lasers als Strahlenwaffe auf harte und weiche Ziele zum Gegenstand hatten. ${ }^{59}$ Am Rande sei noch darauf hingewiesen, dass das BMVg in den 1960er Jahren neben Laser- auch Maserforschungen unterstützte, wie aus einem im April 1964 mit der Firma Rohde \& Schwarz in München geschlossener Forschungsvertrag über die „Erarbeitung der physikalischen Grundlagen eines Rubidium-Masers als Frequenzgenerator höchster Konstanz" hervorgeht, der bei zweijähriger Laufzeit ein Vertragsvolumen von rund 217.000 DM besaß. ${ }^{60}$

Das BMVg förderte jedoch nicht nur Maser- und Laserforschungen im industriellen Bereich, sondern auch an Hochschulen und ihm nahestehenden privatrechtlichen Forschungseinrichtungen in der Bundesrepublik. Im Mittelpunkt der geförderten Projekte standen hier vor allem Untersuchungen der Atmosphäre hinsichtlich ihrer Durchlässigkeit für Laserstrahlen oder, wie im Fall des im April mit der Technischen Hochschule Karlsruhe abgeschlossenen 
Forschungsauftrages für „Mikrowellen-spektroskopische Untersuchungen an Aerosolen", Versuche zur Fernortung chemischer und biologischer Kampfstoffe mittels Mikrowellen. ${ }^{61}$ Eine wichtige Rolle spielte in diesem Zusammenhang die Arbeitsgruppe für Physik der Atmosphäre am Astronomischen Institut der Universität Tübingen, die im Oktober 1961 beim BMVg ein Forschungsvorhaben mit dem Thema „Terrestrische Szintillation Nachthimmel“ beantragte, das dann von Januar 1962 bis Ende 1964 unter dem Titel „Forschungsarbeiten zur atmosphärischen Optik“ von der Hardthöhe mit insgesamt 857.000 DM gefördert wurde. ${ }^{62}$ Wichtig war dieses Forschungsvorhaben für die Bundeswehr vor allem deswegen, weil die Szintillation terrestrischer Lichtquellen die Grenze für optische Lenk- und Beobachtungsverfahren direkt am Boden angab und damit etwa auch für die Nutzung von Laserentfernungsmessern von erheblicher Bedeutung war. ${ }^{63}$ Aus der Arbeitsgruppe des Tübinger Astronomischen Instituts ging später unter weitgehender Kontinuität von Personal und Forschungsprogrammen das nahe bei Tübingen auf Schloss Kressbach untergebrachte, ausschließlich mit Militärforschung beschäftigte Forschungsinstitut für Optik (FfO) der gänzlich vom Verteidigungsministerium finanzierten Forschungsgesellschaft für Angewandte Optik (FGAN) hervor. ${ }^{64}$

$\mathrm{Zu}$ einem weiteren wichtigen Partner des BMVg in der Laserforschung entwickelte sich Ende der 1960er Jahre das Battelle Institut e.V. in Frankfurt am Main. Dieses Institut war im Jahre 1952 als Ableger der amerikanischen Battelle-Stiftung gegründet worden und beschäftigte sich im Rahmen von Auftragsforschungen zunächst mit Fragen der Produkt- und Verfahrensverbesserung sowie der Aufdeckung von Mängelursachen in der Industrie (vgl. Haeske 1977). Seit Ende der 1950er Jahre kamendazu jedoch eigenfinanzierte, langfristig angelegte und zukunftsorientierte Projekte sowie die Übernahme von Aufgaben der öffentlichen Hand, die sich vor allem auf die Bereitstellung von Forschungs- und Entwicklungskapazitäten für das Verteidigungsministerium konzentrierten. ${ }^{65}$ Laserforschungen wurden im Rahmen der zukunftsorientierten Projekte in der Hauptabteilung Physik am BattelleInstitut im Jahre 1962 begonnen, wobei man sich in den Abteilungen für Optik mit Gaslasern und Festkörperlasern sowie in der Abteilung für Grenzflächenforschung mit Injektionslasern beschäftigte. ${ }^{66}$

Zu Beginn des Jahres 1967 waren diese ersten Grundlagenforschungen soweit fortgeschritten, dass das Institut sich fortan verstärkt um Anwendungen seiner Laserentwicklungen kümmern konnte. Die Einstellung des bisherigen Siemens-Laserforschers Karl Gürs als Leiter der Laserentwicklung bei Battelle markiert diese Wende hin zu vor allem militärischen Anwendungen, ${ }^{67}$ deren Hauptaugenmerk zunächst auf der Entwicklung besonders leistungsfähiger Festkörperlaser mit Pulsfolgefrequenzen bis $30 \mathrm{~Hz}$ und mittleren Ausgangsleistungen von einigen Watt lag, die sich auch als Riesenimpulslaser betreiben ließen. „Damit“, so berichtete Karl Gürs 1968, 
„eignet sich der Laser z.B. sehr gut zum Einsatz für Lichtradar“ (Gürs 1968: 19). Im gleichen Jahr wurde am Battelle-Institut „Europas damals stärkster Kohlendioxid-Laser mit einer Leistung von 1,2 kW" in Betrieb genommen (Haeske 1977: 5). Zwei Jahre später war Battelle bereits in der Lage, dem Verteidigungsministerium die bevorstehende Fertigstellung eines 2-kW-GasTransport-Lasers anzukündigen. ${ }^{68}$ Die ausführliche Erläuterung derwichtigen militärischen Anwendungsmöglichkeiten dieses Lasers in einem Exposé unter dem Titel „Militärische Anwendungen des Höchstleistungs- $\mathrm{CO}_{2}$-Lasers“ verband das Institut mit der Mahnung, dass „ein technologischer Rückstand auf diesem Gebiet [...] zu wesentlichen Nachteilen führen“ könne. Um dem vorzubeugen, hieß es weiter in dem Exposé, „schlagen wir die Entwicklung entsprechender Laser vor. Dies kann im Rahmen der Zusammenarbeit zwischen Dornier-System und Battelle geschehen. Ein entsprechender Arbeitsvorschlag liegt dem BMVg bereits vor". ${ }^{69}$ Als erster Schritt wurde darin die Entwicklung eines 10-kW-Dauerlasers innerhalb von anderthalb Jahren für etwa 700.000 DM empfohlen. Die sich daran anschließende Entwicklung eines 100-kW-Lasers sollte noch einmal drei Jahre dauern und etwa drei Millionen DM kosten. 1970 errichtete das Battelle-Institut unter der Leitung von Gürs schließlich eine eigene Abteilung für Laseroptik, die sich schwerpunktmäßig mit militärischen Laserforschungen beschäftigte.

Eine bedeutende Rolle für die Koordination und verwaltungsmäßige Abwicklung der Laserprojekte des BMVg spielte die Fraunhofer-Gesellschaft (FhG) in München (Trischler 2007). So liefen die Anträge der Arbeitsgruppe für Physik der Atmosphäre beim Astronomischen Institut der Universität Tübingen im Jahre 1961 über die FhG an das Verteidigungsministerium in Bonn. ${ }^{70}$ Zwei Jahre später fungierte Professor Reinhard Mecke vom Fraunhofer-Institut für Elektrowerkstoffe in Freiburg - einem jener sechs FhGInstitute, deren Grundfinanzierung wesentlich vom BMVg getragen wurden als Vertreter der Hardthöhe bei den Sitzungen der neu gebildeten Working Group on the Application of Quantum Electronics der NATO. ${ }^{71}$ Als weiterer deutscher Vertreter gehörte dieser Arbeitsgruppe noch der Freiburger Professor Frank Matossi an, der im Juli 1963 über die Aufgabenstellung der Working Group berichtete:

The Group would have to study the different types of lasers. [...] It would have to examine the deficiencies of present lasers and their possible remedies (Mosaics of semi-conductor lasers for example). The Group would also have to decide whether it would merely study the applications of masers and lasers to the identification of aircraft, or extend its investigations to quantum clocks. ${ }^{72}$

Der regelmäßigen Zusammenarbeit auf dem Lasergebiet, insbesondere dem Austausch von Informationen über nationale Entwicklungen innerhalb der NATO, dienten neben der Working Group auch andere Verbindungen, wie der direkte Kontakt des Referats T VI 6 im BMVg zuPartnern aus den USA, etwa dem Frankford Arsenal der US-Streitkräfte oder dem Aeronautical Research 
Laboratory auf Wright Airfield, im Jahre 1962 in der Frage der Laserentfernungsmesser zeigt. ${ }^{73}$ Besondere Bedeutung beim Informationsaustausch im Bereich der Laser-E-Messer kam allerdings der bereits erwähnten Group of Experts on Optical Instruments zu, die regelmäßig zu Sitzungen in Paris zusammenkam. Sie geben einen interessanten Einblick in die Aktivitäten und Fortschritte der Laserentfernungsmessung in den einzelnen NATO-Staaten. So konnten beispielsweise die BritenEnde 1963 einen ersten funktionsfähigen Laser-E-Messer für ihren Panzer Chieftain vorstellen, der bei einer Frequenz von 6 Hertz auf eine Reichweite von 1.000 bis 3.000 Metern eine Genauigkeit von \pm 25 Metern erzielte. Einig war sich die Expertengruppe allerdings darin, dass die Messgenauigkeit auf \pm 10 Meter zu steigern war. Auch die USA kündigten für Mitte Januar 1964 Tests mit neu entwickelten Laser-E-Messern für ihre Panzer an. In Belgien, Frankreich und der Bundesrepublik liefen zu dieser Zeit gleichfalls Entwicklungsarbeiten, während Kanada und Dänemark nach eigenen Angaben keine derartigen Forschungen betrieben. ${ }^{74}$ Sechs Monate später berichtete der BMVg-Vertreter von der Sitzung der Expertengruppe, dass auch Italien Versuche über Laser-E-Messer unternähme. Überall würde es sich allerdings noch um Versuchsmodelle oder Vorstudien handeln, über deren endgültige Tauglichkeit noch nicht entschieden werden könne, „wenn auch die Aussichten dafür günstig“ erschienen. ${ }^{75}$

Dass an den Tagungen der NATO-Sachverständigengruppe für optische Instrumente auch Vertreter der an den jeweiligen nationalen Laserentwicklungen beteiligten Firmen teilnahmen, geht aus einem Antrag des Referates $\mathrm{T}$ VI 6 des BMVg zur Ausstellung von NATO-Ausweisen für zwei Tagungen in Paris im April 1966 an die Vertretung der Bundesrepublik bei der NATO hervor. Vorgesehen waren diese Ausweise für Frank Früngel, der einen Vortrag über neue Ergebnisse auf dem Gebiet der Ausbreitung der Laserstrahlen halten sollte, sowie für Vertreter der optischen Unternehmen Leitz in Wetzlar, Schott in Mainz sowie Zeiss in Oberkochen. ${ }^{76}$ Vor allem die beiden letztgenannten Firmen, die beide zur Unternehmensgruppe Carl Zeiss West Germany gehörten, hatten in den letzten Jahren ihre Aktivitäten in der militärischen Laserforschung erheblich ausgebaut und sollten dabei dem Konkurrenten Leitz bald die Position streitig machen.

\section{Militärische Laserforschung in der bundesdeutschen Industrie: Das Beispiel Carl Zeiss Oberkochen}

Natürlich interessierte sich auch das traditionsreiche optische Unternehmen Carl Zeiss in Oberkochen für die Möglichkeiten einer engen Kooperation auf dem Lasergebiet mit dem BMVg. Die Voraussetzungen dafür schienen bei einem Unternehmen, das bereits im Bereich der klassischen optischen 
Entfernungsmessung nach dem Basisprinzip für die Bundeswehr als Ausrüster tätig war und über jahrelange, intensive Kontakte zur Hardthöhe verfügte, durchaus günstig zu sein. Nur wenige Monate nachdem bei Zeiss Anfang 1962 im Physiklabor der erste Laser arbeitete, ergriff daher der Leiter der damaligen Abteilung für Verteidigungsgeräte des Unternehmens, Johannes Erasmus die Initiative und versuchte Zeiss, in der Entwicklung von Laser-Entfernungsmessern mit dem BMVg ins Geschäft zu bringen.Den äußeren Anlass dafür bot die auf der Jahrestagung der Arbeitsgemeinschaft für Wehrtechnik seitens des zuständigen Referatsleiters für Optik und Feinmechanik der Abteilung für Wehrtechnik des BMVg geäußerte Befürchtung,

dass die langen Entwicklungs- und Lieferzeiten der Firma Zeiss für optische Entfernungsmesser auf dem Basisprinzip möglicherweise dazu führen könnten, dass neuartige Entwicklungen des Auslandes, insbesondere der Amerikaner auf dem Gebiet des „LASER“, die Fertigung der optischen Entfernungsmesser überholen und damit die für diese Fertigung aufgebrachten geistigen und materiellen Investitionen in ihrer Rentabilität wesentlich schmälern könnten. ${ }^{77}$

Erasmus deutete an, dass Zeiss diese Befürchtung des Ministeriums teile und sich deshalb frage, „ob es richtig ist, für die Weiterentwicklung von BasisEntfernungsmessern [...] noch Kapazitäten freizumachen und zu binden“. Als Alternative schlug er dem BMVg vor, Überlegungen darüber anzustellen, „ob man nicht auch der Firma Zeiss einen größeren Forschungs- oder Entwicklungsauftrag für einen optischen Entfernungsmesser nach dem Prinzip LASER übertragen sollte“ ${ }^{78}$ Hinweise auf das bereits funktionstüchtige Versuchsmodell des Festkörperlasers im „Phys-Lab“, die Erfahrungen der Firma bei der Herstellung und Bearbeitung künstlicher Rubine sowie die enge Zusammenarbeit des Unternehmens,,auf diesem speziellen Gebiet der Optik mit einem Hochschulinstitut "79 unterstrichen die Ernsthaftigkeit dieses Vorschlages an das BMVg.

Aus den eingesehenen Unterlagen des BMVg geht nicht hervor, wie man dort auf das Ansinnen der Firma Zeiss reagierte. 1962 wurde das Unternehmen ganz offensichtlich jedoch nicht mit einem entsprechenden Forschungs- und Entwicklungsauftrag versehen, da dieser, wie gezeigt, an die Hamburger Firma Impulsphysik ging. Dennoch begannen bei Zeiss noch 1962/1963 erste Entwicklungsarbeiten für die militärische Nutzung der Impuls-Entfernungsmessung mittels Laser. Die Firmenleitung hatte entschieden, die Entwicklung der Laser-E-Messung parallel zur herkömmlichen Basis-EMessung zunächst auf eigene Kosten zu betreiben, um so eine langfristige Zukunftsperspektive für diesen wichtigen und einträglichen Produktionsbereich zu haben. ${ }^{80}$ Leisten konnte man sich diese Eigenentwicklung Anfang der 1960er Jahre durchaus, da gerade zu dieser Zeit die ersten neuen LeopardPanzer der Bundeswehr mit dem 1,72 Meter Basis-Turm-Entfernungsmessgerät TEM von Zeiss ausgerüstet wurden, von dem bis 1980 insgesamt rund 4.000 Stück in verschiedenen Variationen gebaut und verkauft werden 
konnten. ${ }^{81}$ Darüber hinaus war es bei Zeiss, wie offensichtlich bei anderen Firmen im Rüstungsbereich auch, immer wieder möglich, unter Umgehung der Vorgaben des Verteidigungsministeriums, Gelder, die für andere militärische Entwicklungen bestimmt waren, sozusagen hausintern umzuleiten und so für die eigenen Laserentwicklungen „fruchtbar“ zu machen. ${ }^{82}$

Die Entwicklungsarbeiten für die militärische Nutzung des Lasers bei Zeiss wurden in der Abteilung für Verteidigungsgeräte (VG) durchgeführt. Diese Abteilung war 1956 nach der Gründung der Bundeswehr zunächst unter der Leitung des ehemaligen Panzerobersten und Ritterkreuzträgers Johannes Erasmus $^{83}$ gegründet worden und wurde 1965 unter Erasmus' Nachfolger, Professor Horst Köhler, in Abteilung Sonderoptik (SO) umbenannt. Mit der Gründung von VG knüpfte Zeiss an Jahrzehnte alte militärtechnische Traditionen an, die durch das Ende des Zweiten Weltkriegs und das bis 1955 geltende Forschungs- und Entwicklungsverbot der Alliierten kaum unterbrochen worden waren. Bis zum Ende des "Dritten Reichs" war die Militärtechnik eine der wirtschaftlich tragenden Säulen des Unternehmens gewesen. Seit Ende der 1950er, vor allem aber in den 1960er Jahren gewann die nun unter den Bezeichnungen „Verteidigungsgeräte“ oder „Sonderoptik“ firmierende Militärtechnik einen Teil dieser alten Bedeutung zurück und entwickelte sich erneut zu einer der gewinnträchtigsten Einnahmequellen des Unternehmens. ${ }^{84}$ Neben Kontinuitäten im Bereich qualitativ hochwertiger Produkte, die etwa in der Basis-Entfernungsmessung oder im Bau militärischer Fernrohre bis in die Zeit vor dem Ersten Weltkrieg zurückreichten, ${ }^{85}$ sorgten dafür auch personelle Kontinuitäten, wie etwa die Berufung des einflussreichen Ex-Offiziers Erasmus zum Leiter der Abteilung VG deutlich macht. Schon bis 1945 hatten bei Zeiss in der alten Abteilung Militärtechnik (MIL) vor allem ehemalige Stabsoffiziere den Kontakt zur Wehrmacht aufrecht erhalten; ein System, an das Zeiss in Oberkochen 1956 offenbar mit Erfolg anzuknüpfen suchte. Eine wichtige Rolle in diesem System der Kontinuitäten spielte auch der Physiker Horst Köhler, der schon vor dem Zweiten Weltkrieg bei Zeiss in Jena in der Militärforschung tätig gewesen war, dann während des Kriegs in Kiel bei der Firma Elektroakustik für die Wehrmacht elektroakustische Forschungen betrieben hatte und ab 1945 erneut bei Zeiss in Jena eine Anstellung fand, die er dazu nutzte, sich das dort noch immer vorhandene militäroptische Know-how anzueignen. ${ }^{86}$ Anfang der 1950er Jahre wurde Köhler schließlich von Zeiss in Oberkochen abgeworben, wohin er ausgestattet mit englischen Passierscheinenzusammen mit seiner Familie im Oktober 1951 gelangte.

In Oberkochen beschäftigte sich Köhler zunächst mit der optischen Systemberechnung photogrammetrischer Objektive, was ihm Gelegenheit gab, nebenbei gleichsam auf Vorrat auch militärisch relevante Systeme für Panzerfernrohre zu berechnen. Als Leiter der Mathematischen Abteilung für Fernrohroptik (Math-F) wirkte Köhler aktiv auch an der 1951/1952 bei Zeiss 
wieder aufgenommenen Entwicklung von Entfernungsmessern mit, die zehn Jahre später im Kampfpanzer Leopard I ihren ersten großen Masseneinsatz finden sollten. 1969 ging aus der Abteilung Math-F die ebenfalls von Köhler (bis 1976) geleiteteMathematische Abteilung für Sonderoptik (Math-SO) hervor, die sich speziell mit der militärisch relevanten Optikrechnung befasste (Köhler 1983: 31). Schon 1956 übernahm Köhler, der von 1954 bis 1964 auch als Leiter der Warengruppe Fernrohre (Fern) bei Zeiss fungierte, in der Abteilung VG unter Erasmus die Leitung des neu geschaffenen, sogenannten V-Fernrohr-Labors, in dem nun jene Panzerfernrohre für die Bundeswehr entwickelt wurden, die Köhler in den Jahren zuvor auf Lager berechnet hatte. ${ }^{87}$

Im Jahre 1962 wurde Horst Köhler als Nachfolger von Johannes Erasmus schließlich Leiter der Abteilung VG und damit bei Zeiss der entscheidende Verantwortungsträger für die militärische Laserentwicklung in den 1960er Jahren. Aufgrund seiner langjährigen Erfahrungen im Umgang mit den militärischen Entscheidungsstellen und seiner vielfältigen persönlichen Verbindungen zum BMVg in Bonn und zum Bundesamt für Wehrtechnik und Beschaffung (BWB) in Koblenz wusste er genauestens Bescheid um die Ausrüstungswünsche und Beschaffungsbedürfnisse der Bundeswehr und suchte deshalb die Forschungs- und Entwicklungstätigkeit seiner Abteilung, die ja nicht nur Laser entwickelte, entsprechend auszurichten. Darüber hinaus verfügte er über ausgezeichnete Kontakte zum akademischen Forschungsbereich vor allem durch seine Verbindung zur Technischen Hochschule Stuttgart, an der er sich 1953 für das Fach Physik habilitiert hatte und deren Honorarprofessor er inzwischen geworden war. $\mathrm{Zu}$ seinen persönlichen Bekannten an der TH Stuttgart zählten Hermann Haken und Heinz Pick, die in den frühen 1960er Jahren mehrfach Zeiss aufsuchten, um das Unternehmen zu größeren Aktivitäten in der Herstellung von Laserkristallen zu bewegen. ${ }^{88}$

Köhler leitete die Abteilung VG beziehungsweise SO bis 1967 und übernahm dann bis zu seiner Pensionierung 1976 die Leitung der neu geschaffenen Wissenschaftlich-Optischen Zentralstelle (WOZ), der die zentrale Betreuung von Forschung und Entwicklung im Gesamtbereich der Optik und speziell die Koordinierung der rechnenden Optik mit anderen Entwicklungsstellen übertragen wurde und der auch die vier Mathematischen Abteilungen von Zeiss angegliedert waren (Köhler 1983: 34). Dass er sich in dieser Funktion auch weiterhin mit militärischen Laserentwicklungen beschäftigte, ist daraus zu ersehen, dass er noch im Januar 1971 im BMVg in Bonn auf Ersuchen des Ministeriums einen Vortrag zum Thema „Überlegungen zur Optik von Hochenergielasern" hielt, in dem es im Wesentlichen um die Abschätzung der optischen Möglichkeiten zum Bau von Strahlenwaffen auf Laserbasis ging. ${ }^{89}$

Die eigentlichen Entwicklungsarbeiten zum Laserentfernungsmesser bei Zeiss begannen auf Anregung Köhlers Ende 1962, Anfang 1963 mit einer Parallelentwicklung im Elektro-Labor der Abteilung für Verteidigungsgeräte (VG-EL) und im Hochfrequenzlabor (HF) der Abteilung für 
Hochfrequenzgeräte unter Siegfried Panzer, einem Hochfrequenzingenieur, der von den AEG-Laboratorien zu Zeiss gekommen war und später als Nachfolger Köhlers selbst von 1967 bis 1969 die Abteilung SO leiten sollte (Köhler 1983: 28, 30). Sinn dieser Konkurrenzentwicklung war es, sozusagen zeitgleich die zwei grundsätzlich verschiedenen Methoden zur Erzeugung hochintensiver Laserblitze mittels Q-Switch (Güteschalter) soweit zur Demonstrationsreife zu führen, dass frühzeitig über die Weiterentwicklung der für den jeweiligen Zweck am besten geeigneten Technologie entschieden werden konnte. Während sich die Arbeitsgruppe im HF-Labor unter Panzer für den Q-Switch mittels Kerrzelle entschied, setzte das VG-EL unter Hugo Heinzmann auf den mechanisch-optischen Güteschalter aus Drehspiegeln und Drehprismen. Neben Heinzmann gehörten der Arbeitsgruppe etwa fünf weitere Mitarbeiter an, unter ihnen der Physiker Karlheinz Simon, der von Köhler 1963 für die Laserforschung bei Zeiss angeworben worden war und 1969 schließlich selbst Leiter der Abteilung SO und damit der militärischen Laserentwicklung bei Zeiss werden sollte. ${ }^{90}$ Ziel der Entwicklungen in beiden Laboratorien war ein Versuchsaufbau, mit dem Laser-Entfernungsmessungen mit einer Genauigkeit von \pm 5 bis 10 Metern möglich sein sollten.

1964 waren beide Arbeitsgruppen soweit, dass ein direkter Vergleichstest in Gegenwart der Geschäftsführung durchgeführt werden konnte. ${ }^{91}$ In einer kleinen Dachgeschosskammer wurden dazu nebeneinander die beiden Versuchsaufbauten aufgestellt, um von dort als Zielobjekt das sogenannte Wasserhaus in etwa 600 Metern Entfernung anzumessen. Beide FestkörperLasersysteme arbeiteten problemlos und auch beide Entfernungsmessungen gelangen zufriedenstellend. Dennoch wurde anschließend entschieden, nur das mechanische Verfahren mit rotierendem Drehprisma oder Drehspiegel des VG-EL für die Laserentfernungsmessung weiter zu verfolgen. Den Ausschlag für diese Entscheidung gab einmal die als problematisch empfundene Hochspannungssteuerung der Kerrzelle des Lasers der HF-Arbeitsgruppe, deren Spannungsimpulse sich störend auf die Elektronik der Impulslaufzeitmessung auswirkten, und zum anderen die, allerdings von der technischen Lösung des Q-Switch völlig unabhängige, von der militärischen Entwicklungsgruppe gewählte, direkte digitale Entfernungsanzeige. Letztere erweckte den Eindruck, quasi per Knopfdruck sofort das gewünschte Ergebnis zu liefern und hinterließ daher bei der Geschäftsführung einen entsprechend nachhaltigen Eindruck.

Die weitere Entwicklung der Laserentfernungsmessung lag damit bei Zeiss ab 1964 endgültig in der Hand der Abteilung für Verteidigungsgeräte beziehungsweise Sonderoptik. Sie war inhaltlich gekennzeichnet durch die Suche nach neuen, wirkungsvolleren Lasermedien, durch Berechnungen und Experimente zur Optimierung der Energieeinstrahlung in die Lasermedien sowie durch die Entwicklung neuer Q-Switch-Technologien, um möglichst schnell ein konkurrenzfähiges Laser-E-Messsystem für die militärische Ausrüstung 
anbieten zu können. ${ }^{92}$ Hauptkonkurrenten auf dem Markt waren die Firmen Eltro in Heidelberg sowie Siemens in München, wobei Zeiss ab Mitte der 1960er Jahre vorübergehend versuchte mit Siemens in der Laser-Entfernungsmessung zu kooperieren. Das von dem Siemens-Wissenschaftler Dieter Röß entwickelte „Röß-Ei“ spielte dabei eine herausragende Rolle, da dieser Reflektor aufgrund seiner geometrischen Beschaffenheit eine besonders optimale Energieeinstrahlung in den Laserkristall ermöglichte und deshalb offenbar zeitweise von den zuständigen militärischen Stellen für ein zu entwickelndes Laser-E-Messsystem favorisiert wurde. ${ }^{93}$ Hauptproblem dieses Reflektors war allerdings, dass er ein kompliziertes und damit teures Herstellungsverfahren bedingte und durch seine besondere Eiform viel Platz beanspruchte. Zeiss versuchte diese Nachteile durch die Entwicklung eines Kugelreflektors zu beheben, gab diesen Versuch aber bald wieder zugunsten der herkömmlichen Zylinderreflektoren auf. ${ }^{94}$ Auch die Verbindung mit Siemens in der Laser-E-Messentwicklung wurde 1968 ergebnislos abgebrochen.

Als „Pfahl im Fleisch“ des Anfang der 1960er Jahre von Zeiss beherrschten militärischen Entfernungsmessermarkts in der Bundesrepublik wurde die Firma Eltro in Heidelberg empfunden, von der Zeiss-Wissenschaftler noch Jahre später berichteten: „Die hat uns Angst gemacht!" ${ }^{\text {95 }}$ Hinter Eltro stand, wie bereits erwähnt, der erfahrene US-Laserhersteller Hughes, der sich über diesen Brückenkopf in der Bundesrepublik einen erheblichen Marktanteil bei den militärischen Lasersystemen sicherte. Für die Hersteller bestand das besondere Problem des Militärmarkts darin, dass es sich dabei um keinen freien Markt handelte, sondern immer nur eine Firma als Ausrüster für ein bestimmtes Waffensystem zum Zuge kam. Dies bedeutete im Gegensatz zum freien Markt, dass man bei militärischen Aufträgen entweder den ganzen Zuschlag erhielt oder leer ausging. Letzterestrat für Zeiss in den späten 1970er Jahren bei der Ausrüstung des Kampfpanzers Leopard II für die Bundeswehr ein, als sich Eltro mit einem Laser-E-Messer von Hughes durchsetzte. ${ }^{96}$

Im Gegenzugbedeutete dieser geschlossene Markt für denHersteller, der sich durchgesetzt hatte, die alleinige Marktführerschaft mit gesicherten Gewinnen für ein Produkt, das noch dazu zumeist mit erheblicher finanzieller Unterstützung des potentiellen Abnehmers entwickelt worden war. Welche wirtschaftlichen Konsequenzen der Erhalt des Ausrüstungsauftragsfür ein Unternehmen haben konnte, verdeutlicht ein einfaches Beispiel aus den 1980er Jahren: Der Laserentfernungsmessteil eines etwa fünf Millionen DM teuren Panzers besaß einen Absolutwert von etwa 40.000 DM. Hinzu kamen für das komplette Zielgerät noch die Kosten für die Kreiselstabilisierung, die Elektronik und den klassischen optischen Beobachtungsteil, womit die Gesamtkosten auf etwa 150.000 DM stiegen. Zumeist wurden diese Grundkomponenten eines Zielsystems jedoch mit weiteren Elementen, wie 
etwa einem Nachtsichtgerät (ca. 150.000 DM) oder Multisensorelementen etwa für Mikrowellen, kombiniert, wodurch die Kosten der Gesamteinheit auf rund 500.000 DM, also etwa zehn Prozent der Gesamtkosten des Waffensystems anstiegen. Im Allgemeinen wurden alle genannten Komponenten des Zielsystems von dem Hersteller geliefert, der dafür das Monopol besaß und das von ihm gelieferte System auch nach der Auslieferung an den Auftraggeber weiter logistischbetreute und wartete. Bei Stückzahlen von etwa 500 bis 2.000 Stück entstand so für das Unternehmen, das sich durchsetzte, ein äußerst lukrativer Markt mit entsprechenden Gewinnen.

Um auf diesem umkämpften Markt bestehen zu können, waren erhebliche Forschungs- und Entwicklungsanstrengungen notwendig. Dafür gab es verschiedeneFinanzierungsmodelle, die von einer kompletten Eigenfinanzierung durch das Unternehmen bis hin zur hundertprozentigen Fremdfinanzierung etwa durch das BMVg reichten. In der Abteilung VG/SO bei Zeiss standen für die Eigenentwicklung jährlich etwa vier bis zehn Millionen DM zur Verfügung. Der Fremdanteil, der fast ausschließlich vom BMVg oder vom BWB stammte, lag bei anfangs zehn bis 15 Millionen, stieg jedoch mit dem Umsatzwachstum der Abteilung auf Werte zwischen zwanzig und dreißigMillionen DM jährlich an. ${ }^{97}$ Nachdem Zeiss 1962 zunächst keinen Forschungs- und Entwicklungsauftrag für die Laser-Entfernungsmessung vom BMVg erhalten hatte, wurden die entsprechenden Arbeiten anfangs eigenfinanziert. Das bereits erwähnte Gemeinschaftsprojekt mit Siemens in der zweiten Hälfte der 1960er Jahre zur Entwicklung eines Laser-E-Messgeräts verfügte allerdings bereits über eine fünfzigprozentige Förderung durch das BMVg. Weitere ZeissLaserprojekte, die teilweise vom BMVg beziehungsweise BWB gefördert wurden, waren die Entwicklung von Neodym-Glaslasern sowie später des $\mathrm{CO}_{2}$-Gaslasers. ${ }^{98}$

Die Entwicklung neuer oder verbesserter Lasertypen begann bei Zeiss im Rahmen der Militärforschung bereits im Jahre 1963, da sich der Wirkungsgrad des Drei-Niveau-Rubinlasers für die gewünschten Leistungen des Laser-EMessgeräts als zu gering erwies. Hinzu kam das Problem der Beschaffung geeigneter Rubinkristalle, die man sowohl in der Bundesrepublik alsauch in der Schweiz und in den USA hinzukaufte, ohne allerdings immer zufriedenstellende Ergebnisse erzielen zu können. ${ }^{99}$ Mit der Entwicklung von Vier-NiveauLasern wie dem Neodym-Glaslaser schien sich hier zunächst eine brauchbare Alternative aufzutun, da die Konzerntochter Schott in Mainz entsprechend hochwertige Gläser produzierte. Das in Mainz zur Herstellung der Gläser verwandte Platinschmelzverfahren hatte allerdings den Nachteil, dass immer wieder geringe Spuren Platin von den Schmelztiegeln in die Gläser gerieten und diese dann schnell unbrauchbar werden ließen. Abhilfe brachte hier letztlich erst der Nd:YAG-Laser, dessen seit 1964 in den USA betriebene Entwicklung bei Zeiss aufgegriffen wurde und 1969 zum ersten eigenen Nd:YAG-Laser in der Abteilung SO führte. Die Kristalle dafür mussten 
allerdings aus den USA bezogen werden, da nur dort entsprechend hochwertiges Material hergestellt wurde.

Andere Entwicklungsarbeiten in der Abteilung VG/SO beschäftigten sich seit 1964 mit dem sogenannten Passiv-Q-Switch mit Hilfe sättigbarer Farbstoffe. Zunächst experimentierte man für den Rubinlaser mit den für seine Wellenlänge von 694 nm besonders günstigen Farbgläsern RG 10 und 780 von Schott in Mainz, die jedoch nach einiger Zeit durch die bei den damals verwendeten Laserrubinen hochgradig inhomogene Filament-StrahlungsQuerschnittsverteilung nach kurzer Zeit lokal zerstört wurden. ${ }^{100}$ Zeitweise suchte man dieses Problem durch die Konstruktion eines Drehrevolvers für mehrfachen Farbglaswechsel zu umgehen, gab diese Entwicklung jedoch auf, als man mit in Alkohol gelösten Farbstoffen ans Ziel gelangte. Das erste, von Zeiss an die Bundeswehr gelieferte Laserentfernungsmessgerät aus dem Jahre 1967 war mit einem solchen Farbstoff-Q-Switch für den Rubinlaser ausgerüstet. Mit Aufkommen der Neodym-Laser wurde die Entwicklung des Passiv-Q-Switch für Rubin abgebrochen. ${ }^{101}$ Beim Neodym-Laser löste man in der Abteilung SO bei Zeiss das Q-Switch-Problem durch eine Kombination von mechanischer Güteschaltung mittels Drehprisma mit einem Passiv-QSwitch zur Verkürzung der Impulse durch Farbfolien der Firma Kodak.

Erhebliche Probleme bei der Entwicklung bereitete den Zeiss-Forschern nach eigenen Angaben die inkonstante Ausgangsleistung der Laserspitzen. ${ }^{102}$ Diese wurde durch kleinste thermisch-mechanische Veränderungen der beiden Planspiegel des Resonators ausgelöst, die die Resonanzbedingungen im Laser veränderten und dabei zu ständig wechselnden Impulshöhen oder gar Aussetzern führten. „Wir hatten wohl“, berichtete 1991 im Rückblick Karlheinz Simon, „die Tatsache nicht hinreichend verinnerlicht, dass es sich bei dem Resonator im Wesentlichen um ein empfindliches Interferometer handelt". ${ }^{103}$ Erst die Verwendung mindestens eines sphärischen Spiegels konnte dieses Dejustierungsproblem beheben.

Der Einbau der in der Abteilung VG/SO entwickelten Rubin-, Neodymund Nd:YAG-Laser in die Turmzielfernrohre von Zeiss erfolgte schrittweise in den Jahren 1967 (Rubin), 1968 (Neodym) und 1970 (Nd:YAG). ${ }^{104}$ Den Auftrag zur Entwicklung des Turmzielfernrohres mit Laser (TZFL) hatte Zeiss offenbar 1964 vom Bundesamt für Wehrtechnik und Beschaffung (BWB) erhalten. ${ }^{105}$ Drei Jahre später gingen die ersten TZFL mit Rubinlaser an die Bundeswehr, ohne dort jedoch in größerem Umfang zum Einsatz zu gelangen. Nach Ansicht ehemaliger Mitarbeiter der Abteilung SO scheute die Bundeswehr damals vor allem das medizinische Risiko des Lasereinsatzes für die Truppen, da vor allem für die Augen erhebliche Verletzungsgefahren bestanden. ${ }^{106}$ Erst der Einsatz augensicherer $\mathrm{CO}_{2}$-Laser sollte hier Abhilfe schaffen.

Erfolgreicher war Zeiss dagegen mit der Lieferung von Laserentfernungsmessern an Zweit- oder Drittländer, wobei man mit 
Kooperationspartnern im Ausland oder als Systemlieferant unter einem anderen Generalunternehmer agierte. In den 1970er Jahren wurden so über eine Partnerfirma beispielsweise die österreichische Armee im Infanteriebereich oder auch als Systemausrüster das Heer in Argentinien mit mehreren Hundert Laser-E-Messgeräten von Zeiss beliefert. Weitere Lieferkontakte bestanden nach Holland, wohin mit Lasern bestückte Universalfernrohrperiskope geliefert wurden, oder auch nach Schweden. ${ }^{107}$

Nach Dafürhalten der verantwortlichen Personen war die Laserentwicklung für ein „Entfernungsmesshaus“ wie Zeiss ein Muss. ${ }^{108}$ Ungeachtet derausgezeichneten Verkaufserfolge in der herkömmlichen Laserentfernungsmessung gerade in den 1960er Jahren mit dem TEM, begriffen die zumeist jungen Wissenschaftler und Ingenieure in der militärtechnischen Entwicklungsabteilung bei Zeiss den Laser von Anfang an als innovatives Element für den Markt der Zukunft. Die Geschäftsführung des Unternehmens und Horst Köhler teilten diese Sichtweise und stellten der Abteilung in erheblichem Umfang Eigenmittel der Firma für ihre Forschungs- und Entwicklungsarbeit zur Verfügung. Darüber hinaus sorgten sie dadurch, dass sie lediglich die groben Entwicklungsziele vorgaben, ihren Mitarbeitern ansonsten aber große Freiräume bei der Durchführung ihrer Projekte ließen, für ein entspanntes und der Eigeninitiative förderliches Arbeitsklima. ${ }^{109}$

Zumindest in der Militärtechnik bei Zeiss schlug sich diese Art der Forschungs- und Entwicklungsarbeit in zahlreichen erfolgreichen Laserentwicklungen seit Ende der 1960er Jahre nieder. Zu ihnen zählten etwa das Panzerzieldoppelfernrohr FER ZD II (1970), der Entfernungsmesser EMES 12 und das Nachtsichtgerät FERO ZD 12 (beide 1971), der EMES 12 mit Nd:YAG-Laser (1973), der Torpedozünder mit Farbstofflaser sowie der FERO ZD 12 und das Winkelperiskop beide mit Gated-Viewing-System sowie Halbleiterlaser (alle 1974). ${ }^{110}$ Zahlreiche weitere Neu- und Weiterentwicklungen folgten in den späten 1970er und 1980er Jahren. Die einzelnen Komponenten der Laserentfernungsmessgeräte wurden dabei zunehmend miniaturisiert und standardisiert, so dass schließlich Ende der 1970er Jahre Lasermodule in die von Zeiss gebauten Zielfernrohre, Periskope und handgehaltenen Laser-EMesser (HALEM) eingebaut werden konnten. Bis Ende der 1980er Jahre wurden insgesamt etwa 1.300 Geräte in verschiedenen Konfigurationen von Zeiss gebaut und ausgeliefert. ${ }^{111}$ Die militärische Laserentwicklung wurde so für Zeiss in Oberkochen zu einem beträchtlichen geschäftlichen Erfolg. Ihr Spin-off-Effekt sollte sich vor allem in den 1980er Jahren in der ebenfalls sehr erfolgreichen Entwicklung von medizinischen Lasergerätezeigen, die personell und technisch unmittelbar aus der militärischen Laserentwicklung bei Zeiss hervorging. ${ }^{112}$ Zusammen verkauften beide Abteilungen „einige tausend Einheiten“, was den langjährigen Leiter der Abteilung SO, Karlheinz Simon, 1991 zu der knappen Bemerkung veranlasste: „Ein schönes Beispiel für den Nutzen der Wehrtechnik“. ${ }^{113}$ 
Der hier gefeierte Erfolg einer zivilen Anwendung der militärischen Lasertechnik in den 1980er Jahren darf aber nicht darüber hinwegtäuschen, dass Zeiss in den 1960er in der Lasertechnik vor allem auf deren militärische Variante in Form der Laserentfernungsmessung setzte. Für Zeiss bestand in der frühen Lasertechnik durchaus eine zivile Alternative in Form der Materialbearbeitung mit Lasern, für die im HF-Labor unter Siegfried Panzer bereits zu Beginn der 1960er Jahre wichtige Grundlagen geschaffen werden konnten. Das Unternehmen entschied sich jedoch für die militärische Option und versäumte damit sich frühzeitig einen Wachstumsmarkt zu erschließen, der seit den 1970er Jahren einen anhaltenden Aufschwung erlebte und dabei wertmäßig den Markt für medizinische Laser stets übertraf. ${ }^{114}$

\section{Die bundesdeutsche militärische Laserforschung in den 1960er Jahren: Ein Fazit}

Kehren wir abschließend zu unseren auf Paul Forman sowie Heymann und Martin-Nielsen zurückgehende Ausgangsfragestellung zurück: War der Laser in der Bundesrepublik Deutschland ein ,artefact of German Cold War culture"? So direkt, wie in den USA sicher nicht! Dafür unterschieden sich die Voraussetzungen für eine militärische Förderung der Laserforschung in der Bundesrepublik zu Beginn der 1960er Jahredenn doch zu erheblich von denen in den USA.

Zunächst einmal standen in der Bundesrepublik für die militärische F\&EFörderung insgesamt deutlich weniger Mittel zur Verfügung als in den USA oder in anderen, nach Bevölkerungszahl und Fläche eher vergleichbaren Staaten, wie Frankreich und Großbritannien (vgl. Abb. 1). Des Weiteren befand sich die Bundeswehr noch bis Mitte der 1960er Jahre in ihrer Aufbauphase, die vor allem von der Beschaffung einer kostspieligen Grundausstattung gekennzeichnet war. ${ }^{115}$ Teure F\&E-Programme, etwa nach dem Vorbild des US-Programms für Hochenergielaser, die, wenn überhaupt, allenfalls auf lange Sicht praktischen Nutzen versprachen, hatten daher in dieser Zeit keine Chance auf eine Förderung durch das BMVg. Drittens existierten in der Bundesrepublik praktisch keine den besonderen Forschungsförderungseinrichtungen und Forschungslaboratorien der US-Streitkräfte vergleichbare Institutionen der Militärforschung, die entsprechende Forschungsprogramme im vergleichbaren Umfang wie in den USA hätten finanzieren oder gar selber durchführen können. Damit eng zusammenhängend bestand, viertens, in der Bundesrepublik kein wissenschaftlich-industriell-militärischer Komplex, der in seiner Größenordnung mitdem der USAvergleichbar gewesen wäre. Vor allem in dem für die naturwissenschaftliche Grundlagenforschung zentralen Bereich der akademischen Forschung an den Hochschulen bestanden in der 
Bundesrepublik nach den Erfahrungen des „Dritten Reichs“ und Zweiten Weltkriegs massiveVorbehalte gegen die Militärforschung (Stamm 1981) Das bedeutete zwar nicht, dass an bundesdeutschen Hochschulen überhaupt keine militärisch relevante Forschung betrieben wurde, diese erreichte aber bei weitem nicht das an nordamerikanischen Hochschulen zur selben Zeit übliche Ausmaß. ${ }^{116}$ Fünftens war der militärisch-taktische Auftrag der Bundeswehr als einer in erster Linie für die Verteidigung des Territoriums der Bundesrepublik konzipierten Armee ein völlig anderer als der der US-Streitkräfte, die im Rahmen etwa der Aufrüstung mit interkontinentalen Raketen oder des sich verstärkenden Engagements der USA in Vietnam vor gänzlich anderen militärischen Problemen standen.

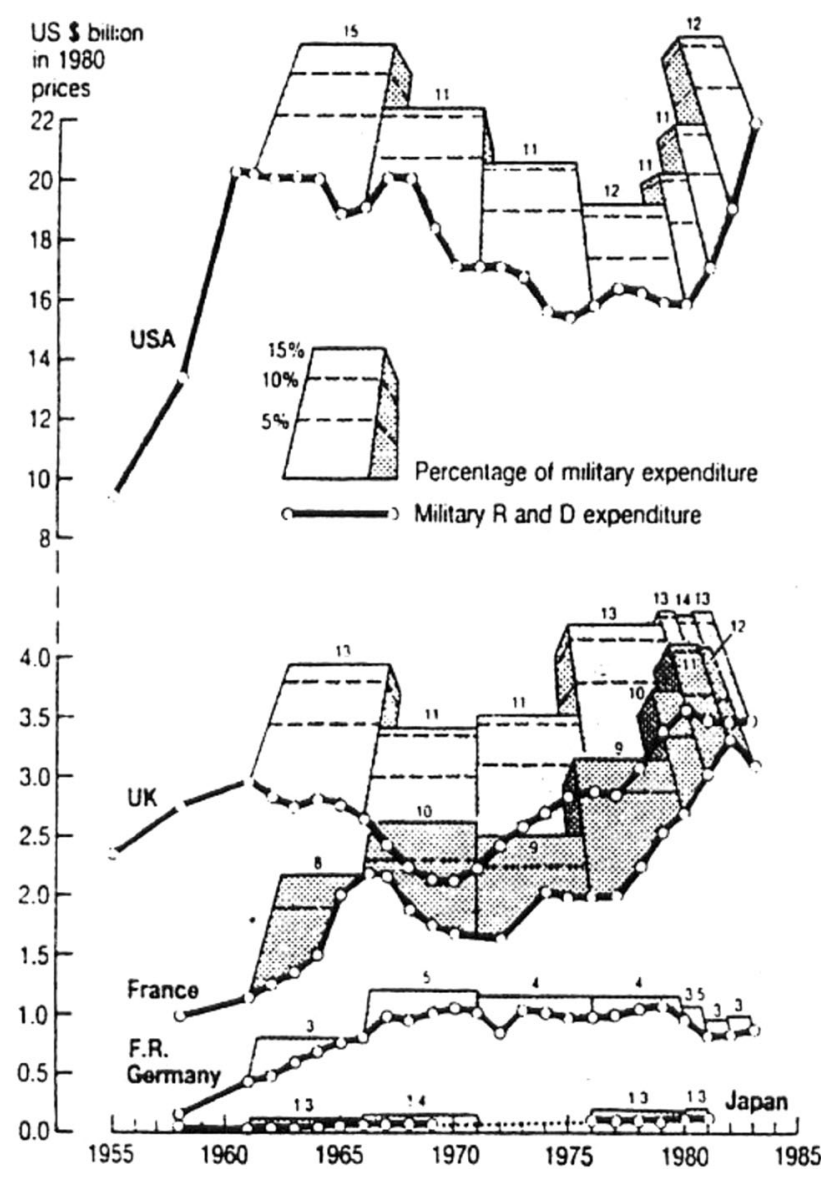

Abb. 1 Militärische F\&E-Ausgaben ausgewählter Staaten in konstanten Preisen von 1980 und als Prozentsatz der gesamten Militärausgaben (Quelle: Gummett 1988: 484) ${ }^{117}$ 
Diese qualitativen und quantitativen Unterschiede hatten zudem zur Folge, dass neben den vergleichsweise kleiner dimensionierten bundesdeutschen F\&E-Aktivitäten im Bereich der Laserforschung auch deren inhaltliche Ausrichtung und Zielsetzung sich in einigen wesentlichen Punkten von denen der USA unterschieden. Das Vorbild der USA, aber auch das Großbritanniens und Frankreichs, vermittelt über die NATO-Expertengruppe für optische Instrumente, spielte als Impulsgeber für den Beginn der militärischen Förderung der Laserforschung und Lasertechnik in der Bundesrepublik in den 1960er durchaus eine wichtige Rolle. Seitens des BMVg wurden in diese Förderung sowohl Unternehmen, außeruniversitäre Forschungseinrichtungen und in geringem Umfang selbst Universitäten einbezogen. Eine Analyse der triple helix des Wechselverhältnisses zwischen Staat, Militär, Wirtschaft und Wissenschaft (Etzkowitz \& Leydesdorff 1997) in der militärischen Forschung und Entwicklung im Laserbereich in der Bundesrepublik zeigt dabei durchaus Ansätze zur Herausbildung eines militärischindustriell-wissenschaftlichen Komplexes mit Unternehmen wie Zeiss in Oberkochen oder Siemens in Münchenbeziehungsweise außeruniversitären Forschungsinstitutionen wie Battelle in Frankfurt, der Früngel $\mathrm{GmbH}$ in Hamburg oder den im militärischen Bereich forschenden Instituten der Fraunhofer-Gesellschaft. Auch die für die Dynamik der Dreifach-Helix wichtigen hybriden Organisationen lassen sich, wie etwa das Beispiel der Arbeitsgruppe Wehrtechnik zeigt, in Ansätzen finden. Einzelne Personen, wie etwa der Leiter der Abteilung Sonderoptik bei Zeiss Horst Köhler, stellten zudem wichtige Schnittstellen zwischen der Industrie auf der einen sowie dem BMVg und/oder Universitäten auf der anderen Seite dar. Beispiele wie die Zusammenarbeit von Leitz und Telefunken in den frühen 1960er Jahren, von Zeiss und Siemens in der zweiten Hälfte der 1960er Jahre sowie von Zeiss mit seinem Tochterunternehmen Schott weisen auf Kooperationsnetzwerke innerhalb der Industrie hin, denen allerdingszumeist wenig Erfolg beschieden war. Nennenswerte kommerzielle Erfolge bundesdeutscher Unternehmen im militärischen Laserbereich vermochten in den 1960er Jahren allenfalls Siemens und Zeiss zu erzielen, wobei gerade das Fallbeispiel Zeiss jedoch auf die unternehmerische Ambivalenz derartiger Erfolge verweist.

Bleibt abschließend die Frage, welche Rolle die militärische Laserforschung oder die Laserforschung und -entwicklung insgesamt in der Bundesrepublik Deutschland in den 1960er Jahren im Kontext der Cold War Science gespielt hat. Für die staatliche Förderung der militärischen Laserforschung und Lasertechnik wie auch für die entsprechenden F\&E-Aktivitäten einschlägiger Forschungseinrichtungen und Unternehmen spielte der Kalte Krieg gerade in den 1960er Jahren eine nicht unwesentliche Rolle. Angesichts des insgesamt bescheidenen Volumens der militärischen Förderung der Laserforschung in der Bundesrepublik hatte dies jedoch kaum nennenswerte Auswirkungen auf die Rüstungsforschung insgesamt. Ein direkter Einfluss der 
Militärforschung auf die zivile Forschung und Entwicklung im Laserbereich lässt sich zwar am Beispiel von Zeiss nachweisen, blieb aber insgesamt bescheiden und mitunter sogar kontraproduktiv. Die bundesdeutschen Universitäten und ihre in der Laserphysik und Lasertheorie durchaus erfolgreichen Forscher hielten sich von einer aktiven Beteiligung an der militärischen Laserforschung aus forschungsethischen und forschungstraditionellen Gründen praktisch völlig fern. Im Gegensatz zu den USA spielten in der Bundesrepublik die universitären Forschungseinrichtungen in der militärischen Laserforschungdamit keine nennenswerte Rolle. Eine wesentlich bedeutendere Rolle für die Entwicklung von Laserforschung und Lasertechnik in der Bundesrepublik Deutschland in den 1960er Jahren spielte dagegen der Knowhow-Transfer über Wissenschaftler wie Wolfgang Kaiser in München oder Hermann Haken in Stuttgart, die um 1960 in den USA direkt in die Anfänge der amerikanischen Laserforschung involviert gewesen sind und nun als Rückkehrer in der Bundesrepublik in den 1960er Jahren eigene universitäre Zentren für Laserforschung aufbauten. Ihre Karrieren und wissenschaftliche Laufbahnen sind letztlich wohl aber auch als ein Produkt der Cold War Science anzusehen.

\section{Anmerkungen}

Bei dem Beitrag handelt es sich um eine überarbeite, stark erweiterte und auf den bundesdeutschen Beitrag zur Lasertechnik konzentrierte Version des Vortrages „Laserforschung und Lasertechnik in Deutschland im Zeitalter des Kalten Krieges - ein Ost-WestVergleich" auf der XIV. Physikhistorischen Tagung der DPG vom 13. bis 15. März 2011 in Dresden. Er basiert auf den Forschungen des Verfassers im Rahmen seiner Habilitationsschrift zur Geschichte von Laserforschung und Lasertechnik in Deutschland in den 1960er Jahren (Albrecht 1997). Zur militärischen Laserforschung in der DDR siehe Albrecht 2012 und Neunhöfer 2001.

1 MASER ist das Akronym für Microwave Amplification by Stimulated Emission of Radiation $=$ Mikrowellenverstärkung durch stimulierte Emission von Strahlung. Maser finden vor allem in der Radioastronomie sowie als Frequenznormale (Atomuhren) Anwendung.

2 Laser ist das Akronym für Light Amplification by Stimulated Emission of Radiation $=$ Lichtverstärkung durch stimulierte Emission von Strahlung.

3 Schawlow erhielt 1981 zusammen mit Nicolaas Bloembergen den Physik-Nobelpreis für den von ihm geleisteten Beitrag zur Entwicklung der Laserspektroskopie verliehen, vgl. Albrecht \& Schulte 2001.

4 Time Magazine vom 2. Januar 1961, S. 40; und New York Time Magazine vom 8. September 1963, S. 27, hier zitiert nach Bromberg 1991: 154f.

5 U.S. News 52 vom 2. April 1962; Reader's Digest 82 (February 1963), S. 97-100, hier zitiert nach Bromberg 1991: 155.

6 Der Spiegel Nr. 16 (1962): 90-92.

7 Vgl. Früngel 1962; Lichtkanone. S. 13; Kießlich-Köcher 1963.

8 Vgl. u. a.: Aladins Lampe. In: Der Spiegel 16 (1962) 16, S. 90-92; Renschick 1963; Reiner 1964; Wunderlampe 1963.

9 Zitiert nach: Der Spiegel 16 (1962) 16, S. 92. 
10 Laser Focus (Januar 1970): 26 f.

11 Laser Focus (Januar 1970): 27.

12 Zur Rolle der Militärforschung an den Universitäten in den USA vgl. Geiger 1992.

13 Vgl. zur allgemeinen Entwicklung der frühen Laserforschung und Lasertechnik in Deutschland Albrecht 1997, 2001. Zur Rolle der DFG in der Förderung der frühen Laserforschung Albrecht 2010. Zur Entwicklung der frühen Laserforschung speziell in der DDR Albrecht 2005, 2007, 2012a, b; sowie Neunhöfer 2001.

14 Vgl. Wehr und Wirtschaft (4/1965): 166.

15 Vgl. Bundesbericht Forschung I,hg. vom Bundesminister für Wissenschaftliche Forschung. Bonn 1965: 14.

16 Vgl. Forschungsbericht IV der Bundesregierung,hg. vom Bundesminister für Bildung und Wissenschaft. Bonn 1972: 58.

17 Zahlenangaben nach den Berichten der Deutschen Forschungsgemeinschaft über ihre Tätigkeit (1963): 58 f.; beziehungsweise (1969): 51-XX.

18 Zusammengestellt nach dem Bundesbericht Forschung I-III. Bonn 1965, 1967, 1969; Forschungsbericht IV. Bonn 1972; Bielfeldt 1977: 50

19 Forschungsbericht IV. Bonn 1972: 58.

20 In der FhG werden heute insgesamt sechs Institute, u.a. die Institute für angewandte Festkörperphysik und für Kurzzeitdynamik in Freiburg oder das Institut für Trieb- und Explosivstoffe in Karlsruhe, überwiegend vom BMVg finanziert,vgl. dazu auch Clauß u. a. 1987: $27 \mathrm{f}$.

21 Vgl. Forschungsbericht IV. Bonn 1972: 59.

22 Bundesbericht Forschung II. Bonn 1967: 79.

23 Vgl. den Kurzbericht über die Dienstreise von Dr. Großkurth (T VI 6) und Dr. Allekotte (T II 3) nach Den Haag vom 13. April 1962, in: Bundesarchiv, Militärarchiv Freiburg (künftig zitiert als: BAMF) BV 3 Nr. 19141: Forschung, Hochfrequenzphysik 1960-1966.

24. Dabei kamen 16 Referenten aus den USA, vier aus Frankreich und je zwei aus Großbritannien und der Bundesrepublik.

25 Vgl. den Kurzbericht vom 13. April 1962, in: BAMF BV 3 Nr. 19141: Forschung, Hochfrequenzphysik 1960-1966.

26 BMVg; Abteilung Wehrtechnik; Unterabteilung Fernmeldetechnik, Elektronik, Feinmechanik, Optik und Akustik; Fachreferat für Optik, Infrarot-, Licht- und Bildtechnik,vgl. dazu die Organisationspläne BAMF ORG 701 (1960) und ORG 800 (1969) des BMVg.

27 BMVg; Abteilung Wehrtechnik; Unterabteilung Wehrforschung; Fachreferat Optik, Infrarot-, Licht- und Bildtechnik, ebd.

28 Kurzbericht vom 13.4.1962, in: BAMF BV 3 Nr. 19141.

29 Anschreiben vom 11.7.1961 mit Bericht vom 22.6.1961, in: BAMF BV 3 Nr. 19141.

30 Schreiben vom 10.11.1961 an das BMVg, in: BAMF BV 3 Nr. 19141.

31 Leitz verpflichtete sich u.a., für Telefunken einen Laser zu bauen, vgl. die Aktennotiz vom 9.1.1962 über die Besprechung in Ulm am 27.11.1961, in: BAMF BV 3 Nr. 19141.

32 Forschungsvertrag Nr. T-214-I-203 - T II 3, unterzeichnet von Leitz am 13.3.1962 und vom BMVg am 23.3.1962, mit Pflichtenheft und Vorkalkulation. Die Vertragssumme sollte in zwei Jahresraten zu 224.530,50 DM (1962) und 200.000 DM (1963) ausgezahlt werden. Leitz sollte das Ergebnis bis zum 31.12.1963 vorlegen, alle Archivalien in: BAMF BV 3 Nr. 19141.

33 Vgl. die Vertragsverlängerung durch das BMVg vom 23.3.1962 sowie den am 22.11.1967 übergebenen Schlussbericht, in: BAMF BV 3 Nr. 19141.

34 Vgl. Forschungsvertrag T-226-I-203 - T II 3, in: BAMF BV 3 Nr. 19145

35 Für die Versuche hatte Leitz aus den USA zwei Rubin-Laserstäbe für zusammen 475 USDollar, zwei Calcium-Fluorid-Stäbe für zusammen 2.500 Dollar und einen $\mathrm{CAWO}_{4}$ Kristall für 1.100 Dollar erworben,Vgl. Anlage I zum Forschungsvertrag T-214-I-203, in: BAMF BV 3 Nr. 19141.

36 Abschlussbericht „Züchtung von Laserkristallen“,undatiert, in: BAMF BW 1 Nr. 39102: Rü VI 4, Versorgungsartikel, Opt. Ziel- und E-Meßgerät, Berichte zu den Studienverträgen 1962-1966. Vgl. dazu auch den „Bericht über die Arbeiten zum Forschungsauftrag Laser-Kristalle“ vom 10. 12.1964, in: BAMF BV 3 Nr. 19145. 
37 Vgl. die Notiz von Ministerialrat Dr.-Ing. Gaertner vom 21.12.1962, in: BAMF BV 3 Nr. 12409: NATO-Dokumente, Sachverständigengruppe für optische Instrumente, Jan.-Juni 1964. Die Tagung der Sachverständigengruppe fand vom 17. Bis 21.12.1962 in Paris statt.

38 Technische Stellungnahme vom 11.7.1963, in: BAMF BV 3 Nr. 19143.

39 Vgl. Aktenvermerk vom 27.6.1962 in: BAMF BV 3 Nr. 19143: Forschung, Hochfrequenzphysik 1961-1966; sowie BV 3 Nr. 19104: Forschung, Hochfrequenzphysik 1960-1966.

40 Schreiben von Dr. Früngel an das BMVg vom 27.4.1962, in: BAMF BV 3 Nr. 19143.

41 Ebd.

42 Vgl. dazu den Aktenvermerk vom 27.6.1962 sowie das Schreiben des BMVg vom 1.10.1962 an die Firma Impulsphysik, mit dem der Forschungsvertrag Nr. T-221-I-203 übersandt wurde. Früngels Kostenvoranschlag für „Nebelauge III“ hatte zunächst laut seinem Schreiben vom 27.4.1962 bei rund 200.000 DM gelegen, in: BAMF BV $3 \mathrm{Nr}$. 19143.

43 Grundsätzlich besteht die Möglichkeit, die Laser-Entfernungsmessung auf zwei Wegen zu realisieren. Bei der technisch einfacheren Lösung des Impulslaufzeitverfahrens wird ein kurzer Laser-Impuls von einem Festkörperlaser ausgestrahlt, der vom anvisierten Objekt reflektiert und von einem Photodetektor am Ausgangsort des Impulses registriert wird. Durch Multiplikation der elektronisch bestimmten Laufzeit des Impulses mit der Lichtgeschwindigkeit und Division mit dem Faktor 2 (Hin- und Rückweg) ergibt sich die Entfernung des Objektes vom Sender. Genauer, aber messtechnisch aufwendiger ist das Verfahren der Phasenvergleichsmessung, bei dem ein kontinuierlicher Laserstrahl eines Gaslasers durch einen elektrooptischen Modulator moduliert wird. Die modulierte Strahlung wird vom Zielobjekt reflektiert und die Phasenverschiebung des reflektierten Lichts gegenüber dem ausgehenden Strahl gemessen, wodurch die Entfernung des Objekts berechnet werden kann,vgl. dazu Molata 1970, Neunhöfer 2001.

44 Am 20.10.1962 erfolgte die Verlängerung bis zum 31.3.1963, und am 26.2.1963 erfolgte die Verlängerung bis zum 30.6.1963, vgl. BAMF BV 3 Nr. 19143.

45 Vgl. den Bericht zum Forschungsvertrag T-221-I-203 „Beiträge zur bildmässigen Entfernungsmessung mit Laser" vom Juli 1963, in: BAMF BV 3 Nr. 19143.

46 Ebd., S. 27.

47 Hinderlich für die Forschung war bislang vor allem die mangelhafte Erschließung der Unterlagen.

48 Vgl. Aktenvermerk vom 27.6.1962, in: BAMF BV 3 Nr. 19143.

49 Vgl. Schreiben vom 22.4.1966, in: BAMF BW 1 Nr. 113818: Rü VI 4, Grüner Laser, 1961-1978.

50 Vgl. BAMF BW 1 Nr. 39103: Rü VI 4, Versorgungsartikel, Opt. Ziel- und E-Meßgerät, Berichte zu den Studienverträgen, 1961-1969.

51 Vgl. BAMF BW 1 Nr. 39104: Rü VI 4, Versorgungsartikel, Opt. Ziel- und E-Meßgerät, Berichte zu den Studienverträgen, 1970-1974.

52 Vgl. ebd.

53 Vgl. das Interview des Verfassers mit Karl Gürs vom 24.1.1994, Laser-Archiv des Verfassers (künftig zitiert als LA).

54 Vgl. den Artikel „Im Kommen: Laser“.Wehrtechnik (10/1977): 56.

55 Vgl. das Schreiben vom 16.12.1963, in: BAMF BW 1 Nr. 113818.

56 Vgl. die Mitteilung vom 10.9.1964 aus dem Zentralinstitut der ELTRO in: BAMF BW 1 Nr. 39101: Rü VI 4, Versorgungsartikel, Opt. Ziel- und E-Meßgerät, Berichte und Gerätebeschreibungen, 1962-1973; sowie BAMF BV 3 Nr. 13554: Studienvertrag: LaserStrahlenschutz, 1964-1967.

57 Vgl. den Artikel „Im Kommen: Laser“.Wehrtechnik (10/1977): 56.

58 Zur Rüstungsproduktion bei MBB vgl. auch Huck u. a. 1989: 66-XX.

59 Vgl. dazu die Artikel des MBB-Mitarbeiters Born 1970, 1976a, b Laserwaffen; und 1976 Militärische Verwendbarkeit. Bis Mitte der 1970er Jahre entwickelte MBB für diese Versuche nach US-Vorbild einen mit chemischer Energie betriebenen gasdynamischen $\mathrm{CO}_{2}$-Hochenergielaser.

60 Vgl. BAMF BV 3 Nr. 3060: T VI, Versorgungsartikel, Fernmeldegeräte und -ausrüstungen, optische Fm.-Geräte für sichtbares u. Unsichtbares Licht, Scheinwerfergeräte für den infraroten Bereich, 1962-1964. 
61 Vgl. BAMF BV 3 Nr. 7861: T II, Forschung, Chemie, 1960-1963. Der genannte Forschungsauftrag war mit ca. 225.000 DM an Fördermitteln ausgestattet.

62 Vgl. Forschungsvertrag Nr. T-213-I-203, in: BAMF BV 3 Nr. 19140: T II, Forschung, Hochfrequenzphysik 1960-1966.

63 Vgl. ebd. den Sachbericht zum Forschungsthema „Forschungsarbeiten zur atmosphärischen Optik“ vom 21.7.1966.

64 Der innerhalb der Arbeitsgruppe im Wesentlichen mit den Laseruntersuchungen beschäftigte Mitarbeiter Dr. D.H. Höhn wurde in den 1970er Jahren Direktor des FfO,vgl. dazu BAMF BW 1 Nr. 55814: Rü VI 4, Berichte des FfO 1962-1974, Bd. 1.

651977 kamen jeweils 35 Prozent der Mittel des Battelle-Instituts vom BMVg und vom BMFT. In den 1960er Jahren dürfte der Anteil des BMVg noch höher gewesen sein, vgl. Haeske 1977: 7.

66 Vgl. Battelle Tätigkeitsbericht 1962. Frankfurt am Main 1963: 33.

67 Vgl. das Interview des Verfassers mit Karl Gürs vom 24.1.1994, LA.

68 Vgl. das Schreiben von Battelle an die Abteilung T VI 6 im BMVg vom 8.1.1970, in: BAMF BW 1 Nr. 57071: Rü VI 4, Battelle-Institut Frankfurt, 1969-1977.

69 Ebd.

70 Vgl. Schreiben der FhG München an die Abteilung T II 3 im BMVg vom 22.10.1961, in: BAMF BV 3 Nr. 19140.

71 Vgl. BAMF BV 3 Nr. 18687: T II, Mitarbeit bei der Nato usw., AC 227: Arbeitsgruppe für die Anwendung der Quantenelektronik.

72 Ebd.: Protokoll der Sitzung der Working Group im NATO-Hauptquartier in Paris am 1.7.1963.

73 Vgl. das Schreiben von T II 3 vom 1.10.1962, in: BAMF BV 3 Nr. 19143.

74 Vgl. das Protokoll des Treffens der NATO-Gruppe in Paris vom 5.12.1963, in: BAMF BV 3 Nr. 12409.

75 Vgl. ebd. den Bericht über die Sitzung am 8. bis 12.6.1964 in Paris vom 13.6.1964.

76 Vgl. das Schreiben vom 28.3.1966, in: BAMF BV 3 Nr. 12412: NATO-Dokumente, Sachverständigengruppe für optische Instrumente, 1966/67.

77 Schreiben an T VI 6 vom 20.4.1962, in: BAMF BV 3 Nr. 19143.

78 Ebd.

79 Es könnte das Institut von Horst Rothe an der TH Karlsruhe gemeint sein, vielleicht aber auch das Physikalische Institut der TH Stuttgart unter der Leitung von Heinz Pick und Hermann Haken.

80 So übereinstimmend Interview mit Horst Köhler vom 8.12.1993 und Karlheinz Simon vom 16.5.1993, beides LA.

81 Vgl. Vortrag Karlheinz Simon, 3 Jahrzehnte Laserforschung und Laserentwicklung bei Zeiss, Oberkochen, dargestellt an ausgewählten Beispielen, 9.7.1991, S. 4, in: Zeiss-Archiv Oberkochen.

82 So jedenfalls die Aussage des ehemaligen Leiters (1969-1991) der Abteilung Sonderoptik (SO) bei Zeiss, vgl. das Interview mit Karlheinz Simon vom 16.5.1993, LA.

83 Erasmus wechselte 1962 von Zeiss in ein Unternehmen der deutschen Schwerindustrie und war später bei der Firma Heidelberger Druckmaschinen tätig. Er gehörte einem illustren Kreis ehemaliger Ritterkreuzträger der Wehrmacht in der bundesdeutschen Wirtschaft an, der unter dem Namen „Mars-Merkur" firmierte, vgl. das Interview mit Horst Köhler vom 8.12.1993, LA.

84 Der Umsatz der "Sonderoptik“ steigerte sich seit den frühen 1960er Jahren von jährlich rund 60 Millionen DM auf schließlich bis zu 270 Millionen DM, die Angaben lt. Interview mit Karlheinz Simon vom 16.5.1993, LA.

85 Vgl. Vortrag Karlheinz Simon, 3 Jahrzehnte Laserforschung und Laserentwicklung bei Zeiss, Oberkochen, dargestellt an ausgewählten Beispielen, 9.7.1991, S. 4, in: Zeiss-Archiv Oberkochen.

86 Köhler, Jahrgang 1913, hatte Physik in Jena studiert und dort 1937 bei Abraham Esau mit einer Arbeit über die Lichtmodulation von Glühlampen promoviert. Diese Arbeit wurde bereits damals von Zeiss gefördert, da das Unternehmen ein Lichtsprechgerät für die Wehrmacht entwickelte. Ab 1937 war er kurz für Zeiss tätig, ehe er 1938 nach Kiel ging,vgl. das Interview mit Horst Köhler vom 8.12.1993, LA.

87 Vgl. dazu das Interview mit Horst Köhler vom 8.12.1993, LA. 
89 Vortrag Horst Köhler, Überlegungen zur Optik von Hochleistungslasern, Manuskript, 12.1.1971, in: Zeiss-Archiv Oberkochen.

90 Vgl. das Interview mit Horst Köhler vom 8.12.1993 und Karlheinz Simon vom 16.5.1993, beides LA; Vortrag Karlheinz Simon, 3 Jahrzehnte Laserforschung und Laserentwicklung bei Zeiss, Oberkochen, dargestellt an ausgewählten Beispielen, 9.7.1991, in: Zeiss-Archiv Oberkochen.

91 Vgl. das Interview mit Horst Köhler vom 8.12.1993 und Karlheinz Simon vom 16.5.1993, beides LA; Vortrag Karlheinz Simon, 3 Jahrzehnte Laserforschung und Laserentwicklung bei Zeiss, Oberkochen, dargestellt an ausgewählten Beispielen, 9.7.1991, in: Zeiss-Archiv Oberkochen.

92 Vgl. das Interview mit Karlheinz Simon vom 16.5.1993, LA; Vortrag Karlheinz Simon, 3 Jahrzehnte Laserforschung und Laserentwicklung bei Zeiss, Oberkochen, dargestellt an ausgewählten Beispielen, 9.7.1991, in: Zeiss-Archiv Oberkochen.

93 Vgl. das Interview mit Horst Köhler vom 8.12.1993 und Dieter Röß vom 6.12.1993, beides LA.

94 Interview mit Karlheinz Simon vom 16.5.1993, LA.

95 Ebd.

96 Ebd. sowie Wehrtechnik (10/1977): 56.

97 Vgl. das Interview mit Karlheinz Simon vom 16.5.1993, LA. Der Umsatz von SO bei Zeiss stieg nach Simon von etwa 60 Mio. DM pro Jahr auf bis zu 270 Mio. DM in den besten Zeiten an. Für das Jahr 1985 wurde der Rüstungsumsatz der Firma Carl Zeiss in Oberkochen in den Betrieben in Baden-Württemberg öffentlich auf 140 Mio. DM bei einem Gesamtumsatz von 960 Mio. DM (ca. 14,6 \%) geschätzt, vgl. Clauß u.a. 1987: 9.

98 Vgl. Material von Karlheinz Simon; LA.

99 Vgl. das Interview mit Karlheinz Simon vom 16.5.1993, LA.

100 Vgl. den Vortrag von Karlheinz Simon, 3 Jahrzehnte Laserforschung und Laserentwicklung bei Zeiss, Oberkochen, dargestellt an ausgewählten Beispielen, 9.7.1991, in: ZeissArchiv Oberkochen.

101 Vgl. das Interview mit Karlheinz Simon vom 16.5.1993, LA.

102 Vgl. den Vortrag von Karlheinz Simon, 3 Jahrzehnte Laserforschung und Laserentwicklung bei Zeiss, Oberkochen, dargestellt an ausgewählten Beispielen, 9.7.1991, in: ZeissArchiv Oberkochen.

103 Ebd., S. 6 f.

104 Angaben nach: Laseraktivitäten bei Carl Zeiss im Bereich Wehrtechnik (Sondertechnik), in: Zeiss-Archiv Oberkochen.

105 Vgl. das Interview mit Karlheinz Simon vom 16.5.1993, LA.

106 Ebd. Mit diesen Angaben korrespondieren die zahlreichen Forschungsaufträge des BMVg für Laserschutzfilter und allgemeine Schutzmaßnahmen gegen Laserlicht in den 1960er Jahren, siehe BAMF BW 1 Nr. 39103; und BV 3 Nr. 13554.

107 Nach Schweden unterhielt der erste Leiter der Abteilung VG, Johannes Erasmus, bereits in den 1950er Jahren enge Kontakte, vgl. das Interview mit Horst Köhler vom 8.12.1993 und Karlheinz Simon vom 16.5.1993, beides LA

108 Vgl. das Interview mit Horst Köhler vom 8.12.1993 und Karlheinz Simon vom 16.5.1993, beides LA.

109 Ebd.

110 Vgl. die Zusammenstellung der Laseraktivitäten der Firma Carl Zeiss im Bereich der Wehrtechnik (Sondertechnik), in: Zeiss-Archiv Oberkochen.

111 Vgl. den Vortrag von Karlheinz Simon, 3 Jahrzehnte Laserforschung und Laserentwicklung bei Zeiss, Oberkochen, dargestellt an ausgewählten Beispielen, 9.7.1991, S. 8, in: Zeiss-Archiv Oberkochen.

112 Die Abteilung „Med“ bei Zeiss übernahm den von SO entwickelten Nd:YAG-Laser mit Farbstoff-Folien-Q-Switch für ihre augenchirurgischen Geräte. Der spätere Leiter dieser Abteilung, Hans-Richard Weinheimer, war zuvor bei SO tätig,vgl. ebd. Vortrag Simon sowie Köhler 1983.

113 Vgl. den Vortrag von Karlheinz Simon, 3 Jahrzehnte Laserforschung und Laserentwicklung bei Zeiss, Oberkochen, dargestellt an ausgewählten Beispielen, 9.7.1991, S. 7, in: Zeiss-Archiv Oberkochen. 
114 Das Gesamtvolumen für Lasersysteme zur Materialbearbeitung betrug weltweit 1988 etwa 1,6 Milliarden DM, was einem Anteil von etwa 4,3\% am Weltmarkt für Lasersysteme entsprach. Der entsprechende Anteil der Medizinischen Lasergeräte lag dagegen nur bei 3,5\%. Der Anteil bundesdeutscher Hersteller am Weltmarkt für Lasersysteme zur Materialbearbeitung, die in den meisten Anwendungsbereichen als technologisch führend angesehen wurden, lag 1988 bei rund $11 \%$, vgl. dazu Reinhard (1990):128.

115 Vgl. den Beitrag „Verteidigungsforschung in der Bundesrepublik“. Wehr und Wirtschaft (4/1965): 166; sowie den Bundesbericht Forschung II,hg. vom Bundesminister für Wissenschaftliche Forschung. Bonn 1967, S. 78.

116 Vgl. dazu die entsprechende Klage von Mennen 1969.

117 Die Grafik verdeutlicht die führende Rolle Großbritanniens und Frankreichs bei den militärischen Forschungsausgaben in Europa in den Jahren 1955 bis 1985, hinter denen die bundesdeutschen Ausgaben deutlich zurück standen.

\section{Literatur}

Albrecht, Helmuth 1997. Laserforschung in Deutschland 1960-1970. Habil.-Schrift Universität Stuttgart.

Albrecht, Helmuth und Uwe Schulte 2001. Nicolaas Bloembergen, Arthur Leonhard Schawlow, Kai Manne, Börje Siegbahn. In: Brockhaus Nobelpreise. Chronik herausragender Leistungen. Mannheim: Brockhaus: 784-785.

Albrecht, Helmuth 2001. Die Innovation des Lasers in Deutschland - Forschungen an der TU Berlin und der Universität in Jena im Vergleich. In: Johannes Abele, Gerhard Barkleit und Thomas Hänseroth (Hg.). Innovationskulturen und Fortschrittserwartungen im geteilten Deutschland (= Schriften des Hannah-Arendt-Instituts für Totalitarismusforschung 19). Köln: Böhlau: 263-276.

Albrecht, Helmuth 2005. Laser für den Sozialismus - Der Wettlauf um die Realisierung des ersten Laser-Effekts in der DDR. In: Susan Splinter u. a. (Hg.). Physica et historia. Festschrift für Andreas Kleinert zum 65. Geburtstag (= Acta Historica Leopoldina 45). Stuttgart: Wissenschaftliche Verlagsgesellschaft: 471-491.

Albrecht, Helmuth 2007. Laserforschung an der Friedrich-Schiller-Universität in Jena. In: Uwe Hoßfeld, Tobias Kaiser und Heinz Mestrup (Hg.). Hochschule im Sozialismus. Studien zur Geschichte der Friedrich-Schiller-Universität Jena (1945-1990). Köln: Böhlau: 1436-1469.

Albrecht, Helmuth 2012a. Innovation im Zeichen von Planwirtschaft und SED-Diktatur. Die Anfänge der Entwicklung der Laser-Technologie in Jena in den 1960er Jahren. In: Klaus Dicke (Hg.). Die Rolle der Universität in Wirtschaft und Gesellschaft (= Texte zum Jenaer Universitätsjubiläum 7). Jena: Garamond: 171-201.

Albrecht, Helmuth 2010. The German Research Foundation and the Early Days of Laser Research at West German Universities during the 1960s. In: Helmuth Trischler und Mark Walker (Hg.). Physics and Politics. Research and Research Support in Twentieth Century Germany in International Perspective (= Beiträge zur Geschichte der Deutschen Forschungsgemeinschaft 5). Stuttgart: Franz Steiner: 161-195.

Albrecht, Helmuth 2012b. Militärische Laserforschung in der DDR in den 1960er Jahren. In: Uwe Fraunholz und Sylvia Wölfel (Hg.). Ingenieure in der technokratischen Hochmoderne. Thomas Hänseroth zum 60. Geburtstag (= Cottbuser Studien zur Geschichte von Technik, Arbeit und Umwelt 40). Münster: Waxmann 2012: 241-266.

Bender, Hans 1962. Todesstrahlen noch außerhalb der technischen Möglichkeiten. Soldat und Technik (5/10): 526-529.

Bielfeldt, Carola 1977. Rüstungsausgaben und Staatsintervenismus. Das Beispiel Bundesrepublik Deutschland 1950-1970. Frankfurt am Main: Campus.

Bericht der Deutschen Forschungsgemeinschaft über ihre Tätigkeit 1963. Bad Godesberg 1963. Bericht der Deutschen Forschungsgemeinschaft über ihre Tätigkeit 1969. Bad Godesberg 1969. Born, Gunthard K. 1970. Laserwaffen. Laser und angewandte Strahlentechnik (2): 37-38. 
Born, Gunthard K. 1976a. Laserwaffen - sind sie realisierbar? Wehrtechnik (7): 22-25.

Born, Gunthard K. 1976b. Militärische Verwendbarkeit des Lasers. Wehrtechnik (12): 73-76.

Bromberg, Lisa 1991. The Laser in America 1950-1970. Cambridge/Mass.: MIP Press.

Bundesbericht Forschung I. Hrsg. vom Bundesminister für Wissenschaftliche Forschung. Bonn 1965

Bundesbericht Forschung II. Hrsg. vom Bundesminister für Wissenschaftliche Forschung. Bonn 1967

Bundesbericht Forschung III. Hrsg. vom Bundesminister für Wissenschaftliche Forschung. Bonn 1969

Bundesbericht Forschung IV. Hrsg. vom Bundesminister für Wissenschaftliche Forschung. Bonn 1972

Forschungsbericht IV der Bundesregierung, hg. vom Bundesminister für Bildung und Wissenschaft. Bonn 1972

Clauß, Winfried u. a. 1987. Rüstungsindustrie in Baden-Württemberg. Tübingen: Verein für Friedenspädagogik.

Ebeling, D. 1963. Modulation von Impulslasern. Physikalische Verhandlungen (14, Heft 9/10): 163-164.

Etzkowitz, Henry und Loet Leydesdorff (Hg.) 1997. Universities and the Global Knowledge Economy. A Triple Helix of University-Industry-Government Relations. London: Pinter.

Fabian, Cornelia 2012. Technologieentwicklung im Spannungsfeld von Industrie, Wissenschaft und Staat. Zu den Anfängen des Innovationssystems der Materialbearbeitungslaser in der Bundesrepublik Deutschland 1960 bis 1997. Diepholz: Verlag GNT.

Forman, Paul 1987. Behind Quantum Electronics: National Security as Basis for Physical Research in the United States, 1940-1960. Historical Studies in Physical and Biological Sciences (18): 149-229.

Forman, Paul 1992. Inventing the Maser in Postwar America. In: Arnold Thackray (Hg.). Science After'40 (= Osiris 7): 105-134.

Fritsch, Michael und Louis F. Medrano Echalar 2015. New Technology in the Region Agglomeration and Absorptive Capacity Effects on Laser Technology Research in West Germany, 1960-2005. Economics of Innovation and New Technology (24/1-2): 65-94.

Früngel, Frank 1962. Todesstrahlen. Umschau 17: 551.

Früngel, Frank 1963. Laserblitze in der High-Speed Photography. Proceedings of the Sixth International Congress on High-Speed Photography: S. 52-56.

Früngel, Frank 1963. Neues aus der Kurzzeitphotogtraphie. Umschau 63/13: 408-409.

Geiger, Roger L. 1992. Science, Universities, and National Defense, 1945-1970. In: Arnold Thackray (Hg.). Science After'40 (= Osiris 7): 26-48.

Gugel, Günther u. a. 1986. Rüstung und Wirtschaft. Tübingen:Verein für Friedenspädagogik.

Gummett, Philip 1988. The Government of Military R\&D in Britain. In: Everett Mendelsohn, Merritt Roe Smith und Peter Weingart (Hg.). Science, Technology and the Military (Sociology of the Sciences XII/2). Dordrecht: Kluwer: 481-506.

Gürs, Karl 1968. Der Laser und seine Anwendung. Battelle-Information (1): 6-19.

Haeske, Horst 1977. 25 Jahre Battelle-Institut e.V. 25 Jahre Auftragsforschung. BattelleInformation (26): S. 2-9.

Heymann, Matthias und Janet Martin-Nielsen 2013. Introduction: Perspectives on Cold War Science in Small European States. Centaurus. An International Journal of the History of Science and its Cultural Aspects (55): 221-242.

Hounshell, David A. 2001. Rethinking the Cold War; Rethinking Science and Technology in the Cold War; Rethinking the Social Study of Science and Technology. Social Studies of Science (31): 289-297.

Huck, Burhardt J. u. a. 1989. Rüstungsindustrie und Rüstungskonversion in der Region München. Firmen, Produkte, Beschäftigte und Perspektiven für die 90er Jahre. Frankfurt am Main: Campus.

Kießlich-Köcher, H. 1963. Strahlen gegen Raketen? Militärwesen (7/2): 243-250.

Köhler, Horst 1983.30 Jahre Forschung und Entwicklung im Zeiss Werk Oberkochen. Band 1. Oberkochen (maschinenschriftliches Dokument, LA).

Laser Focus (Januar 1970). Newtonville/Mass. 
Mendelsohn, Everett, Merritt Roe Smith und Peter Weingart 1988. Introductory Essay Science and the Military: Setting the Problem. In: dies. (Hg.). Science, Technology and the Military (Sociology of the Sciences XII/1). Dordrecht: Kluwer: XI-XXIX.

Molata, A. 1970. Anwendungen des Lasers. Wehrtechnik (5): 209-215.

Neunhöfer, Reinhard 2001. Jenaer Lasertechnik zwischen Wissenschaft, Wirtschaft und Staatssicherheit. Phil. Diss. Universität Stuttgart.

Reinhard, Michael 1990: Stand und wirtschaftliche Perspektiven der industriellen Lasertechnik in der Bundesrepublik Deutschland. München 1990 (ifo studien zur industriewirtschaft 39).

Reiner, R. 1964. Aladins moderne Lampe. Draht und Welle (14/5): 10-11.

Renschick, UDO 1963. Laser, ein Wunderwerkzeug der Technik. Automatisierung (8/10): 32-34.

Schall, R. 1969. 20 Jahre Nato. Die heutige Zusammenarbeit im Bündnis in der Verteidigungsforschung und Technik. Wehrtechnik (4): 125-129.

Schawlow, Arthur L. 1961. Optical Masers. Scientific American (204/6): 52-61.

Seidel, Robert W. 1987: From Glow to Flow. A History of Military Laser Research and Development. Historical Studies in the Physical and Biological Sciences (18): 111-147.

Seidel, Robert W. 1988: How the Military Responded to the Laser. Physics Today (41): 12-19.

Stamm, Thomas 1981. Zwischen Staat und Selbstverwaltung. Die deutsche Forschung im Wiederaufbau 1945-1965. Köln: Verlag für Wissenschaft und Politik.

Trischler, Helmuth 2007. Das Fraunhofer-Institut für Angewandte Festkörperphysik im Kontext der bundesdeutschen Forschungs- und Innovationsgeschichte: Ein zeithistorischer Essay. Freiburg: Fraunhofer-Institut für Angewandte Festkörperphysik: 29-66.

Trudeau, A. G. 1962. Research for Survival. American Society of Civil Engineers. Transactions (127/ 5): 20-39.

Weiss, Gert 1962. Lichtlawine aus dem Laser. Wehr und Wirtschaft (6): 299-300.

Wunderlampe der Neuzeit: Der Laser. Universum (18/7): 334-336.

\section{Helmuth Albrecht}

Institut für Industriearchäologie, Wissenschaftsund Technikgeschichte (IWTG)

TU Bergakademie Freiberg

Silbermannstr. 2

09599 Freiberg

Germany

E-mail: helmuth.albrecht@iwtg.tu-freiberg.de 